\title{
Hsp90 as a Gatekeeper of Tumor Angiogenesis: Clinical Promise and Potential Pitfalls
}

\author{
J. E. Bohonowych, U. Gopal, and J. S. Isaacs \\ Department of Cell \& Molecular Pharmacology, Hollings Cancer Center, Medical University of South Carolina, \\ 86 Jonathan Lucas Street, Charleston, SC 29425, USA \\ Correspondence should be addressed to J. S. Isaacs, isaacsj@musc.edu
}

Received 25 December 2009; Accepted 12 April 2010

Academic Editor: Sundaram Ramakrishnan

Copyright ( $\odot 2010$ J. E. Bohonowych et al. This is an open access article distributed under the Creative Commons Attribution License, which permits unrestricted use, distribution, and reproduction in any medium, provided the original work is properly cited.

\begin{abstract}
Tumor vascularization is an essential modulator of early tumor growth, progression, and therapeutic outcome. Although antiangiogenic treatments appear promising, intrinsic and acquired tumor resistance contributes to treatment failure. Clinical inhibition of the molecular chaperone heat shock protein 90 (Hsp90) provides an opportunity to target multiple aspects of this signaling resiliency, which may elicit more robust and enduring tumor repression relative to effects elicited by specifically targeted agents. This review highlights several primary effectors of angiogenesis modulated by Hsp90 and describes the clinical challenges posed by the redundant circuitry of these pathways. The four main topics addressed include (1) Hsp90-mediated regulation of HIF/VEGF signaling, (2) chaperone-dependent regulation of HIF-independent VEGF-mediated angiogenesis, (3) Hsp90dependent targeting of key proangiogenic receptor tyrosine kinases and modulation of drug resistance, and (4) consideration of factors such as tumor microenvironment that pose several challenges for the clinical efficacy of anti-angiogenic therapy and Hsp90-targeted strategies.
\end{abstract}

\section{Introduction}

The concept of antiangiogenic targeting as a means to suppress malignancy came to the forefront of cancer therapeutics in the early 1970s, based upon the pioneering work of Folkman [1]. Tumor vascularization is a critical component of cancer progression, malignancy, and metastasis. As rapidly dividing cancer cells reach a critical tumor size, the mass outgrows its supply of blood, oxygen, and nutrients. Continued tumor growth depends upon the ability of microenvironmental stressors to trigger the activation of a complex and intricately interconnected signaling network that culminates in vascularization of the growing tumor. This activation process is essential for initiation of the "angiogenic switch," a rate limiting event of tumor progression. Subsequent tumor vascularization culminates in either enhanced angiogenesis, the sprouting from local vessels, or vasculogenesis, the formation of neovessels through bone marrow-derived cell (BMDC) recruitment. Realization that the angiogenic switch may represent a universal Achilles heel for all tumors, coupled with elucidation of druggable targets within this network, has invigorated the field of antiangiogenic therapy, resulting in a rich pipeline of therapeutic compounds [2].

\section{Emergence of Hsp90 Inhibitors as Antiangiogenic Chemotherapeutic Agents}

Although a number of antiangiogenic therapies are presently in clinical use, the vast majority of these target a specific molecule or receptor family [3]. Despite a relatively high degree of specificity, the clinical efficacy of these therapies as curative agents remains poor. Though initial responsiveness may occur, the ultimate outcome is treatment resistance due to drug-dependent selection of intrinsic and adaptive resistance mechanisms. Therefore, attention has turned to chemotherapeutics targeting heat shock protein 90 (Hsp90), which simultaneously target multiple proangiogenic regulators, and may thus weaken the signaling resiliency characteristic of tumor cells. The prototypic Hsp90 inhibitor geldanamycin (GA) demonstrates potent antiangiogenic and antitumorigenic properties $[4,5]$. At the molecular level, Hsp90 plays a critical role in the proper folding of its 
client or substrate proteins [6] and also serves as a scaffold protein to facilitate interactions between several receptor tyrosine kinases (RTKs) and their substrates. Therefore, Hsp90 inhibitors are unique as antiangiogenic agents in that they regulate the activity of hundreds of proteins, many of which support cancer growth [7-9]. In addition, Hsp90 expression is increased in many cancers, allowing sustained activation of cancer-specific dysregulated pathways and the buffering of stress conditions characteristic of the tumor microenvironment [10]. As a result, the evaluation of emergent Hsp90 inhibitors is a current focus of drug discovery efforts across multiple cancers $[8,9,11]$.

The first discovered Hsp90 inhibitor, GA, is a naturally occurring benzoquinone ansamycin [12] that acts as a nucleotide mimetic to inhibit ATP-dependent Hsp90 chaperone activity. Although useful as a research tool, the pharmacological liabilities of GA prohibit its clinical use [13] leading to the development of subsequent generations of Hsp90 inhibitors including the GA analog 17-(allylamino)17-demethoxygeldanamycin (17-AAG) and its water soluble version 17-dimethylaminoethylamino-17-demethoxygeldanamycin (17-DMAG), both of which are being evaluated in multiple Phase I, II, and III clinical trials [1416]. Alternatively, Hsp90 function and angiogenesis may be impaired by histone deacetylase (HDAC) inhibitors, that act upon the chaperone in a manner distinct from compounds that target its N-terminal ATP-binding pocket. Currently, the HDAC inhibitor LBH589/Panobinostat is under evaluation in Phase I and II trials [17]. The development of Hsp90 inhibitors, although in its infancy a decade ago, is now coming to the forefront of cancer therapy, with over 13 new entities being tested in a variety of preclinical models and human trials [18]. The antiangiogenic and antitumor effects of these agents will be discussed herein.

\subsection{Rationale for Hsp90-Dependent Targeting of HIF.} Hypoxia inducible factor (HIF) is perhaps one of the most potent proangiogenic proteins regulated by Hsp90. Of the three known HIF isoforms, HIF-1 and HIF-2 contribute to cancer progression and their widespread overexpression in cancers correlates with increased mortality [19]. During tumor growth, HIF transactivates genes to favor survival under conditions of decreasing oxygen and nutrient availability [20]. A substantial number of these genes, such as vascular endothelial growth factor (VEGF) (Figure 1), fall within the category of proangiogenic cytokines, which collectively tip the scales in favor of angiogenesis and neovascularization as part of the angiogenic switch. Activation of this HIF-driven angiogenic switch releases growth constraints upon the tumor and sustains subsequent progression. Tumor cells have evolved multiple mechanisms for upregulating HIF expression and activity, most commonly via modulation of the HIF- $\alpha$ subunit, normally a tightly regulated labile protein subject to proteasomal degradation [21-23]. In general, these mechanisms block oxygen-dependent prolyl hydroxylase (PHD) enzymes from tagging HIF for degradation, ultimately preventing its proteasomal degradation via the von Hippel Lindau (VHL) ubiquitin ligase. VHL can also be inactivated via genetic mutation, genetic loss, or epigenetic suppression, all events most commonly associated with hereditary and sporadic clear cell renal cell carcinoma (CCRCC) $[24,25]$. HIF overexpression correlates with highly vascularized tumors, resistance to chemo- and radiotherapy, and overall poor prognosis [26]. The essential role of HIF in tumor angiogenesis has been functionally validated in many models $[27,28]$, highlighting its importance and validity as a clinical target.

Although no specific anti-HIF targeting strategies have been approved, a number of currently utilized antiangiogenic agents have suppressive effects upon HIF activity or synthesis and diminish HIF-mediated VEGF expression [19]. Several of these agents exhibit antiangiogenic and antitumorigenic effects in preclinical models [29-32] and two specific HIF inhibitors are being evaluated in Phase I trials, the small molecule PX-478 [33], and the antisense oligonucleotide EZN-2968 [34]. The use of Hsp90 inhibitors as a strategy to target HIF emerged shortly after HIF was first identified as an Hsp90 client protein [35]. Many of the antivascular effects of Hsp90 inhibitors are likely due to the ability of this class of inhibitors to downregulate HIF activity. We and others have shown that both the HIF- $1 \alpha$ and HIF- $2 \alpha$ subunits are client proteins for Hsp90 and that chaperone activity is required for HIF stability and function [35-39]. Importantly, Hsp90 inhibitors, such as GA and its clinical derivative 17-AAG, promote proteasomal degradation HIF- $\alpha$ in a PHD/VHL and oxygen-independent manner, instead of utilizing the ubiquitin ligase RACK1 [37, 40-42]. Thus, Hsp90 inhibitors abrogate HIF signaling even in the absence of a functional PHD/VHL system, suggesting that these pharmacological agents may be able to dampen the constitutive HIF signaling associated with most solid tumors. This notion is supported by the ability of Hsp90 inhibitors to decrease VEGF secretion from cancer cells, impair endothelial cell tubule formation in vitro, and reduce in vivo tumor size and vascularization [43-45].

\subsection{Hsp90 as an Effector of VEGF Expression and Angiogenic} Activity. Although HIF is a main effector of VEGF-mediated signaling, HIF-independent proangiogenic processes also contribute to increased VEGF-dependent proangiogenic signaling [46]. $\mathrm{NF} \kappa \mathrm{B}$ potently induces tumor vascularization, in part through its ability to upregulate VEGF expression through an IL-8/NF $\kappa$ B signaling axis [47] (Figure 1). Secretion of IL-8, a potent proangiogenic factor, is mediated through a variety of mechanisms including other cytokines, cellular stress, and hypoxia (Figure 1) [48]. A positive feedback loop has also been reported whereby $\mathrm{NF} \kappa \mathrm{B}$ can induce IL-8 and angiogenin secretion to promote tumor neovascularization through the recruitment of bone marrow derived cells [49]. Blockade of $N F \kappa B$ signaling in an orthotopic model of ovarian cancer inhibited tumor growth, reduced tumor angiogenesis, and suppressed VEGF and IL-8 expression [50]. In addition to its role in HIF-independent upregulation of VEGF, $\mathrm{NF} \kappa \mathrm{B}$ has also been shown to transcriptionally induce the physiological expression of HIF1 [51]; however, it is not known whether this pathway may also exist in transformed cells. Although NF $\kappa$ B itself is not a validated Hsp90 client protein, multiple direct 


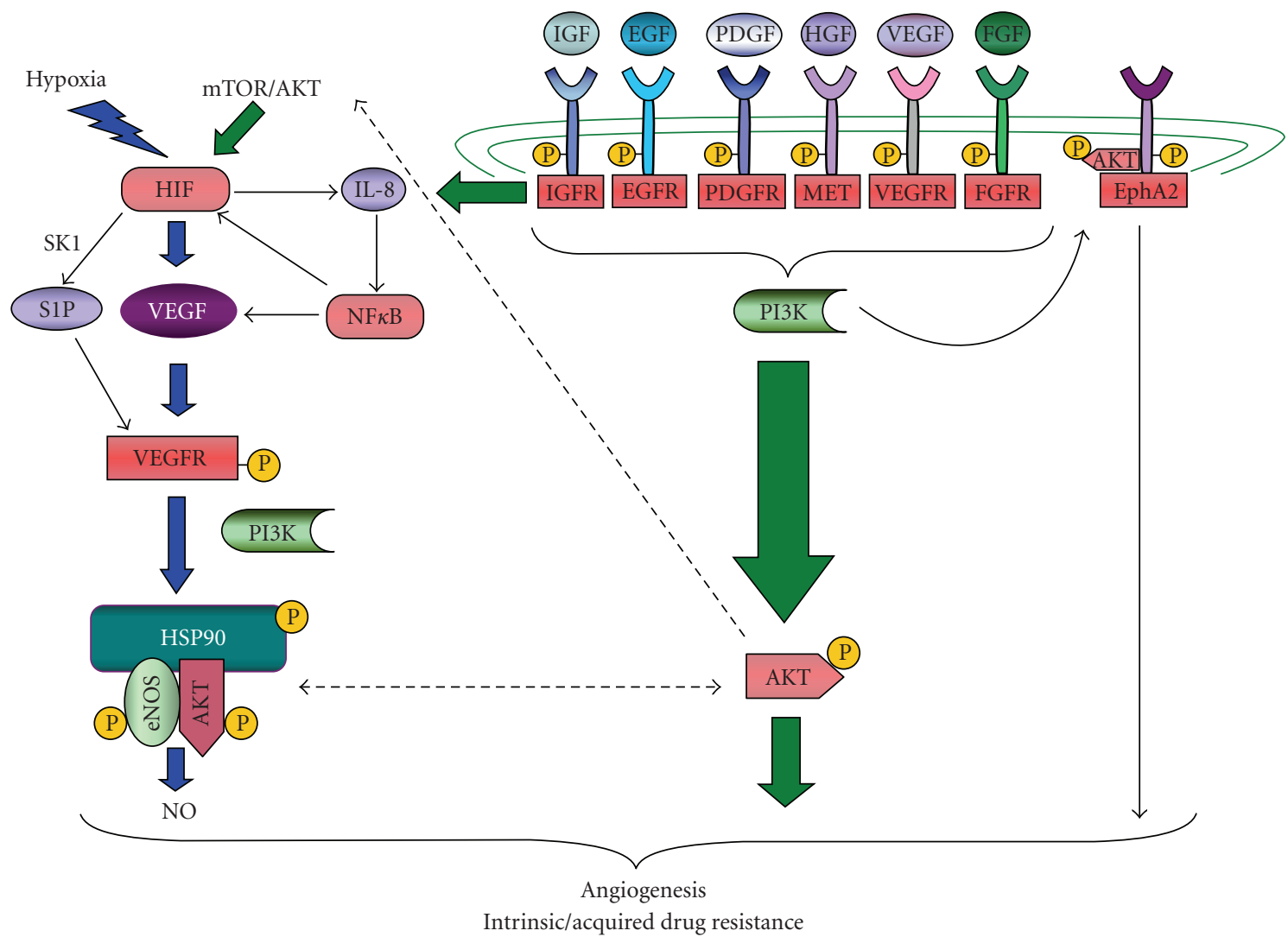

FIGURE 1: Hsp90-dependent modulation of proangiogenic signaling pathways in cancer. Hsp90 regulates multiple arms of angiogenic signaling in cancer. Key signaling molecules that are either direct clients or indirectly modulated by Hsp90 are shaded in red. One pathway that is commonly upregulated during tumorigenesis is the HIF/VEGF signaling axis. Tumor hypoxia and other stimuli induce HIF expression and subsequent activity, leading to a cascade of events that reinforce VEGF expression and angiogenesis. Importantly, several key mediators of this pathway, including HIF and VEGFR, are dependent upon Hsp90 for their function. As indicated, RTK activation also potently upregulates HIF via AKT/mTOR -mediated translation. RTKs additionally transactivate EphA2, a recently identified Hsp90 client protein known to participate in tumor vascularization. Providing another level of complexity, HIF also upregulates the expression of several RTK ligands (e.g., HGF and TGF-alpha), as well as RTK receptors (EGFR, IGFR), thereby reinforcing these signaling networks. Hsp90 additionally plays a role in NFאB-dependent VEGF expression and regulates downstream effectors of VEGF signaling, including AKT-mediated eNOS phosphorylation. Given the intertwining levels among Hsp90 and angiogenic signaling cascades, Hsp90 intervention is predicted to impair signaling at many levels within these redundant pathways, with the overall effect of suppressing tumor angiogenesis.

modulators of NFkB activation are subject to regulation by Hsp90, permitting Hsp90-inhibitor-mediated suppression of $\mathrm{NF} \kappa \mathrm{B}[52-55]$. Moreover, $\mathrm{NF} \kappa \mathrm{B}$ may directly regulate Hsp90 expression through two binding sequences in the Hsp90 promoter, [56] suggesting the possibility of a feedback loop whereby Hsp90 inhibition decreases $\mathrm{NF} \kappa \mathrm{B}$ activity, which may further decrease Hsp90 levels. It remains to be seen whether these HIF-independent angiogenic regulators of VEGF are appropriately suppressed upon Hsp90 inhibitor treatment in relevant preclinical models.

In addition to its involvement in both HIF and $\mathrm{NF} \kappa \mathrm{B}$ mediated VEGF production, Hsp90 also modulates downstream effectors of VEGF-dependent signaling. VEGF mediates many of its proangiogenic effects through stimulation of the enzyme endothelial nitric oxide synthase (eNOS). In endothelial cells, activated eNOS leads to nitric oxide (NO) synthesis and release [57], which in turn promotes angiogenesis through endothelial cell proliferation and migration, as well as having effects on blood flow through modulation vascular tone and permeability [58,59]. Mechanistically, VEGF binds to and activates the VEGF-R2 receptor, promoting phosphorylation of associated Hsp90 [60]. This phosphorylated Hsp90, in turn, serves as a scaffold to facilitate the association between eNOS and AKT, thereby promoting AKT-dependent phosphorylation and activation of eNOS (Figure 1) [61]. Microenvironmental factors such as hypoxia may facilitate angiogenesis by increasing the interaction between Hsp90 and eNOS concomitant with increased AKT activity in endothelial cells [62]. The association between eNOS and Hsp90 can be further stabilized by sphingosine-1-phosphate (S1P), a bioactive lipid induced during hypoxia in an HIF-dependent manner [63, 64], and reciprocally, S1P may stimulate HIF activity [65] and elicit chemoresistance [66]. Given that VEGFR, AKT, eNOS, and $\mathrm{NF} \kappa \mathrm{B}$ share a dependence upon Hsp90, [67, 68] (Figure 1), Hsp90 inhibitors have the potential to target 
multiple steps of this pathway, as demonstrated by the potent suppression of VEGF and NO release both in vitro $[69,70]$ and in preclinical models $[71,72]$. Additionally, 17-AAG directly inhibits transcription of eNOS mRNA in the in vitro angiogenic HUVEC (human umbilical vein endothelial cells) model, although the mechanism remains unknown [71]. Moreover, Hsp90 inhibitors decrease the expression of activated AKT and eNOS in HUVEC cells, concomitant with inhibitory effects upon tubule formation [72]. Therefore, the antiangiogenic properties of Hsp90 inhibitors are due in part to their ability to suppress HIFdependent and independent VEGF expression and dampen the signaling potential of VEGF through modulation of VEGFR, AKT, and eNOS function. In light of these functions, Hsp90 chemotherapeutics have the potential to potently suppress tumor angiogenesis by attenuating the secretion of cancer-derived angiogenic factors as well as by blocking paracrine and autocrine signaling in the tumor-associated endothelium.

2.3. Hitting Hard: Hsp90 Inhibition as a Multifaceted Strategy to Combat Tumor Vascularity and Drug Resistance. Although VEGF plays a pivotal role in tumor angiogenesis and VEGFtargeted agents represent the cornerstone of many antiangiogenic approaches in malignant disease, the majority of these initially responsive tumors subsequently acquire drug resistance [3]. Broadly speaking, antiangiogenic tyrosine kinase inhibitors (TKIs) act by neutralizing VEGF/VEGFR signaling, VEGFR in combination with other proangiogenic RTKs, or target distinct RTKs that may not include VEGFR $[3,73,74]$. Common among all TKI-initiated strategies is a lack of durable response, an outcome that represents the biggest clinical challenge with TKI therapy. This universal property of therapeutic failure is a product of both de novo resistance, due to the inherent genetic complexity and heterogeneity of tumors, and to acquired resistance, a result of the plasticity and signaling redundancy characteristic of tumor cells [3, 75-77]. In the case of failure of VEGF-targeted therapy, the activation of compensatory mechanisms fosters adaptation and independence from VEGF signaling [78]. Compensatory and redundant signaling is a characteristic of several cancer types, such as glioblastoma multiforme (GBM), wherein activation of PDGF, MET, and EGFR family members collectively limits the therapeutic efficacy of specific TKI agents [79]. Importantly, the ability of angiogenic cytokines to activate their cognate RTK receptors is also a major determinant in conferring chemoresistance. Therefore, the salient finding that RTKs comprise the largest category of Hsp90 client proteins [7, 68, 80] (Figure 1) holds clinical promise for the ability of Hsp90-directed agents to suppress angiogenic signaling and overcome therapeutic resistance in diverse cancers. The following section will discuss Hsp90-modulated effectors of RTK driven angiogenesis, highlight several redundant signaling mechanisms contributing to drug resistance, and outline prospects for Hsp90 intervention and opportunities for reversal of this resistance.

The PDGF-FGF-VEGF signaling module represents a highly integrated pathway commonly activated in a number of cancers. The PDGF- $\alpha$ receptor participates in cell transformation, regulation of vascular permeability, and VEGF expression [81, 82]. Highlighting the complexity and interconnectedness of angiogenic signaling, PDGF also synergizes with FGF to stimulate neovascularization [83] and FGF, in turn, cooperates with VEGF to stimulate blood vessel maturation and function [84]. Clinically, several antiangiogenic TKIs are currently approved as inhibitors for these growth factor/RTK pairs, such as imatinib mesylate (gleevec), sorafenib, and others [85]. The interconnectedness of these pathways suggests a potential benefit of combining TKIs in antiangiogenic therapy, supported by the clinical observation that combination of a pan-VEGFR inhibitor (sunitinib) with a PDGF inhibitor (AGO13736) delayed tumor progression more effectively in cytokine refractory renal cancer [86]. The EGF-IGF-HGF-VEGF signaling module represents another proangiogenic molecular hub. Activation of the MET RTK receptor by its ligand, hepatocyte growth factor (HGF), which may be upregulated by a HIFdependent pathway [87], has pleiotropic angiogenic effects including upregulation of VEGF and its receptor [88], as well as stimulation of HIF-mediated VEGF secretion [89]. MET also cooperates with epidermal growth factor receptor (EGFR) through the downstream effectors PI3K/AKT [77]. EGFR family members EGFR and HER2 are overexpressed in many cancers and are integral to tumor progression, in part due to their ability to stimulate release of angiogenic factors including VEGF [90]. Continuing the theme of crosscommunication, EGFR and HER2 cooperate with IGFR through the PI3K pathway to synergistically increase vessel growth [91]. Although cancer cells harboring amplified MET are initially sensitive to MET TKIs in vitro, they evade this inhibition, despite durable MET inhibition, via reactivation of EGFR and downstream mediators [92]. Antiangiogenic TKIs targeting these receptors are being evaluated in Phase I and II clinical trials [3].

The targeting of EGFR with gefitinib provides a wellcharacterized scenario illustrating the pleiotropic mechanisms at play in drug evasion. One common mechanism for therapeutic escape is conferred by secondary mutations in RTKs that impair the ability of targeted therapeutics to recognize and block receptor function. For example, although at least three EGFR TKIs are in clinical trials (imatinib, gefitinib, and erlotinib), resistance occurs with a frequency of $70 \%$ in lung cancer patients, due to the acquisition of secondary mutations in the kinase domain $[76,93]$. Another caveat is that gefitinib treatment results in the activation of signaling pathways that collectively serve to limit its antitumor activity and allow acquired resistance to emerge. A number of these resistance mechanisms have been identified in both preclinical and clinical studies and include activation of oncogenic signaling, HGF, VEGF, and PI3K/AKT activation, the latter of which plays a role in de novo resistance and sustenance of EGFR activation in a number of cancers [77, 85, 94-98]. A number of studies implicate the contribution of IGF-1R and FGFR as participants in both intrinsic and acquired resistance to EGFR TKIs [98]. These findings strongly advocate the use of combination therapy as a means to overcome this signaling redundancy and subsequent drug resistance. In 
support of this notion, it was demonstrated that coadministration of gefitinib and an IGF-1R inhibitor (NVPAEW541) in a gefitinib resistant xenograft model reversed drug resistance [99]. Other reports also document the importance of cotargeting downstream molecules, including the Hsp90 regulated effectors $\mathrm{PI} 3 \mathrm{~K} / \mathrm{mTOR}$ and MEK, in regaining drug responsiveness in EGFR mutant cancers [98]. These examples highlight the large scope of compensatory mechanisms utilized by tumor cells and the need for broad acting agents and/or combinatorial approaches such as those represented by Hsp90-directed chemotherapeutics.

The inherent ability of Hsp90-targeted agents to suppress multiple proangiogenic receptors and effector proteins bodes well for their prospects in overcoming the redundancy of signaling required for adaptive responses. In support of this premise, geldanamycins (GAs) destabilize MET, inhibit its angiogenic function [100], and suppress coordinate signaling effectors that elude receptor-specific targeting strategies [92]. Blocking Hsp90 also inhibits IGFR and PDGFR function and reduces their angiogenic potential in vivo $[45,101]$. Similarly, GAs suppress EGFR/HER2 signaling, impair multiple pathways associated with receptor upregulation, and reduce tumor growth and vascularization in vivo $[45,102$, 103]. Importantly, TKI resistant EGFR mutant receptors are exquisitely sensitive to Hsp90 inhibitors and these agents synergize with EGFR inhibitors [104]. Interestingly, clinical administration of 17-AAG led to tumor regression in HER-2 positive metastatic breast cancer patients [105], demonstrating the oncogene-addicted nature of this tumor. The basis for the poor response in HER-2 negative subtypes remains unknown, given that $\mathrm{Hsp} 90$ inhibition also cotargets pivotal downstream effectors of RTK signaling, such as FAK, MAPK, and AKT $[67,106,107]$, which would be expected to amplify their suppressive effects and thwart adaptive responses. This ability of Hsp90 inhibition to fundamentally alter network circuitry in tumor cells bodes well for their ability to potentiate the efficacy of targeted or cytotoxic drugs administered in tandem, a notion supported by its synergistic antitumor and antivascular in numerous in vitro and preclinical models [104, 108-112].

\section{EphA2 Receptor as a Conduit for RTK Activation and a Driver of Angiogenesis}

The Eph RTK superfamily is of recent interest in relation to proangiogenic proteins targeted by Hsp90-directed therapy. Several of the 16 Eph RTKs demonstrate functions pertaining to vessel development during embryogenesis and in cancer [113]. In particular, the EphA2 receptor is emerging as a pivotal regulator of physiological and pathological angiogenesis. EphA2 plays an essential role in developmental angiogenesis, as null endothelial cells fail to undergo cell migration and vascular assembly both in vitro and in vivo [114]. A definitive role for EphA2 in malignancy is illustrated by diminished tumor growth, angiogenesis, and metastasis in EphA2 deficient mice [115]. Furthermore, EphA2 is commonly detected in the tumor associated vasculature, where it facilitates angiogenesis [116-118]. EphA2 is overexpressed in a number of human malignancies, particularly in highly vascular
GBM tumors, where it serves as a prognostic factor [119]. Unlike most other ligand/RTK interactions, the association of ephrinA1 ligand with the EphA2 receptor is inhibitory, due to subsequent internalization and proteasomal degradation of the receptor $[113,120]$. In cancers, ligand expression is downregulated during the malignant process [119], leading to constitutive EphA2 signaling. In terms of therapeutic options, no clinical TKIs against EphA2 have yet been developed, nor have any already approved clinical agents been shown to target EphA2. However, targeting of EphA2 signaling by either siRNA-mediated suppression of receptor expression [121] or administration of a selective antibody [122] are approaches that have demonstrated efficacy in preclinical models. Highlighting an alternative therapeutic approach, we found that EphA2 receptor activity requires Hsp90 function [123] and, further, that Hsp90-targeting agents interfere with EphA2-mediated signaling and promote receptor destabilization. Therefore, Hsp90 inhibition may represent a therapeutic approach to neutralize receptor function and reduce the aggressiveness of EphA2-driven cancers.

In keeping with the theme of cross-communication, EphA2 is earning its place as an essential member of an expanding network of protumorigenic effectors. First, EGFR activation drives MAPK signaling, which leads to its upregulation in a number of aggressive cancers [124]. Secondly, EGFR family members co-opt EphA2 to promote cell motility and proliferation $[115,125]$. Third, EphA2 also cooperates with VEGFR, as demonstrated by its requirement for VEGF-induced endothelial cell migration and tubule formation [126]. Finally, EphA2 function is transduced by its ability to complex with AKT, the latter of which is activated by diverse RTK ligands (EGF, FGF, HGF, and PDGF) [120]. This multifaceted mechanism for EphA2 activation reinforces the theme of the previous section in that the effective blockade of EphA2 signaling will require the simultaneous cotargeting of multiple RTKs. Growth factor-mediated AKT phosphorylation in turn leads to AKT-dependent EphA2 phosphorylation, which facilitates EphA2/AKT complex formation (Figure 1). Formation of this signaling unit is critical for tumor cell migration and invasiveness. Although it remains unclear how this pathway mechanistically translates to angiogenic potential, AKT is a known mediator of angiogenic processes [127] and AKTmediated EphA2 phosphorylation within GBM-associated tumor vasculature increases with malignancy [120]. Given that AKT is a validated Hsp90 target protein [128] activated in a number of malignancies, and that AKT acts as a conduit for EphA2 signaling, Hsp90-targeting strategies should be effective in blocking EphA2/AKT-dependent angiogenesis. Interestingly, most of the aforementioned angiogenic cytokines are also reported to stimulate VEGF secretion, due in part to their ability to stimulate HIF translation via mTOR, a downstream effector for AKT. As recently reviewed [19], many of the aforementioned TKIs also downregulate HIF translation. Collectively, cytokine-mediated activation of RTKs, and subsequently of AKT, stimulates HIF translation, thereby potentiating VEGF-dependent signaling and sustained angiogenesis (Figure 1). In a recent twist, 
it was shown that HIF-2 regulates both the expression and activation of multiple RTKs [129]. Furthermore, the suppression of HIF dramatically improves tumor responses to Sunitinib in colon cancer cells and strikingly prolonged complete responses in half of the tumor bearing mice [130]. This demonstrated ability of HIF to regulate RTK signaling and potentiate the effects of antivascular agents further emphasizes the complexity and cross-pollination of these signaling pathways and supports the rationale for utilizing Hsp90-targeted agents as a strategy to cotarget HIF proteins and proangiogenic RTKs.

\section{Murky Waters: Clinical Challenges of Hsp90 Inhibition}

Although Hsp90 sustains a multitude of angiogenic processes critical for cancer progression, and Hsp90-targeted agents demonstrate favorable responses across multiple cancers in preclinical models, they have fared less well in the clinic. Recent clinical failures include the use of 17-AAG in advanced prostate cancer and CCRCC [131, 132], the latter a particularly surprising outcome given the putative HIF-dependent and angiogenic nature of this tumor. However, these patients had already presented with advanced metastatic disease, and it is therefore unclear whether improved responses might have been observed with earlier intervention. This section will evaluate some of the more complex issues and potential pitfalls that may offer insight into the variable clinical response of these inhibitors, including pharmacologic considerations, undesired effects of Hsp90-directed therapy, and role of the tumor microenvironment.

4.1. Toxicity, Metabolism, and Delivery. Discordance between Hsp90-targeted efficacy in preclinical models compared with less favorable clinical outcomes may be due to a number of pharmacologic factors, independent of the ability of these agents to target the appropriate protumorigenic pathways. One issue may pertain to drug formulation, as 17-AAG/Tanespimycin is a substrate for the multidrug resistance (MDR) transporter P-glycoprotein, and the related MRP efflux pump [133]. Acquired resistance of cells via this mechanism has been observed in cell culture [134]. Another caveat is that drug potency requires reduction by NAD $(\mathrm{P}) \mathrm{H}$ :Quinone Oxidoreductase I (NQO1) [133, 135, 136]; yet information is lacking on NQO1 expression profiles in treated patients. A new generation of purine-based Hsp90 inhibitors was subsequently developed [137] whose activity does not depend upon this reduction event [134]. Purinebased compounds are currently at the forefront of Hsp90 inhibitor advancement with several derivatives exhibiting increased potency and decreased toxicity when compared to 17-AAG [138]. Furthermore, these agents are not subject to metabolism by NQO1/DT-diaphorase enzymes nor to efflux by P-glycoprotein, and tumor cells in culture that have acquired resistance to 17-AAG remain susceptible to these newer agents [134, 139]. Therefore, despite disappointing early clinical results with 17-AAG, clinical enthusiasm for the next generation of Hsp90 inhibitors remains high.
4.2. Molecular Caveats of Hsp90 Inhibition. In addition to drug formulation challenges, inhibition of cellular Hsp90 initiates a heat shock response [140] that triggers activation of heat shock factor 1 (HSF-1) [140] and corresponding upregulation of prosurvival chaperones Hsp27, Hsp70, and Hsp90. This heat shock response antagonizes drug potency in vitro and in vivo [140-143]. Furthermore, HSF-1 expression is essential in supporting malignant transformation [144]. A heat shock response is similarly initiated with the newer purine derivatives $[139,142]$ and therefore represents a characteristic associated with this general class of Hsp90 targeting agents. However, it may be possible to address this undesired effect through combination treatments. For example, cisplatin suppresses 17-AAG-mediated HSF-1 activation and synergistically promotes tumor cell death in vitro [145]. It remains to be seen whether clinical modulation of the HSF1-mediated heat shock response may enhance the clinical efficacy of Hsp90-directed therapy.

Another less well-understood consideration involves molecular factors that may alter the efficiency of Hsp90mediated client destabilization. Many Hsp90 client proteins are destabilized via the concerted action of ubiquitin ligases and the proteasomal pathway; however little is known about the molecular effectors involved in client destabilization following Hsp90 inhibition. Taking HIF as an example, we have previously demonstrated that the association of HIF with its dimerization partner ARNT promotes dissociation of Hsp90 from HIF, with resultant protection from Hsp90 inhibiting agents [40]. Since that report, others have documented additional HIF binding or accessory proteins that may share a similar propensity to modulate the efficiency of Hsp90 directed HIF degradation [42, 146, 147], highlighting the previously unappreciated molecular complexity associated with Hsp90-targeted therapy. Recently, a group demonstrated that expression of Cullin5, a ubiquitin ligase, was required for optimal degradation of HIF and ErbB2 [148]. Importantly, Cullin5 expression is decreased in an overwhelming majority of breast cancers [149], suggesting that tumor cells may acquire the ability to evade or limit the destabilization effects of Hsp90-dependent chemotherapy upon subsets of clients. The identification of these modifiers and an analysis of their expression in human cancers may be required to gauge the clinical potential of Hsp90-directed therapy against specific clients or signaling pathways.

\subsection{Fighting a Tiger: Contribution of Stroma to Tumor} Angiogenesis and Malignant Progression. Another factor in the disconnect between preclinical results and efficacy in patients is likely attributed to the influence of the tumoradjacent stromal tissue. The tumor stroma, which influences the microenvironment surrounding the neoplastic lesion, is a driving force of both tumor growth and vascularization. This heterogenous stroma is comprised of a variety of cell types, including fibroblasts/myofibroblasts, smooth muscle cells and endothelial cells, immune cells, and bone marrow progenitor cells (Figure 2) [150], all of which collaborate to spur tumor growth. Clinically, an abundant fraction of the tumor reactive stroma is composed of cancer-associated fibroblasts (CAFs), or myofibroblasts, whose presence is 


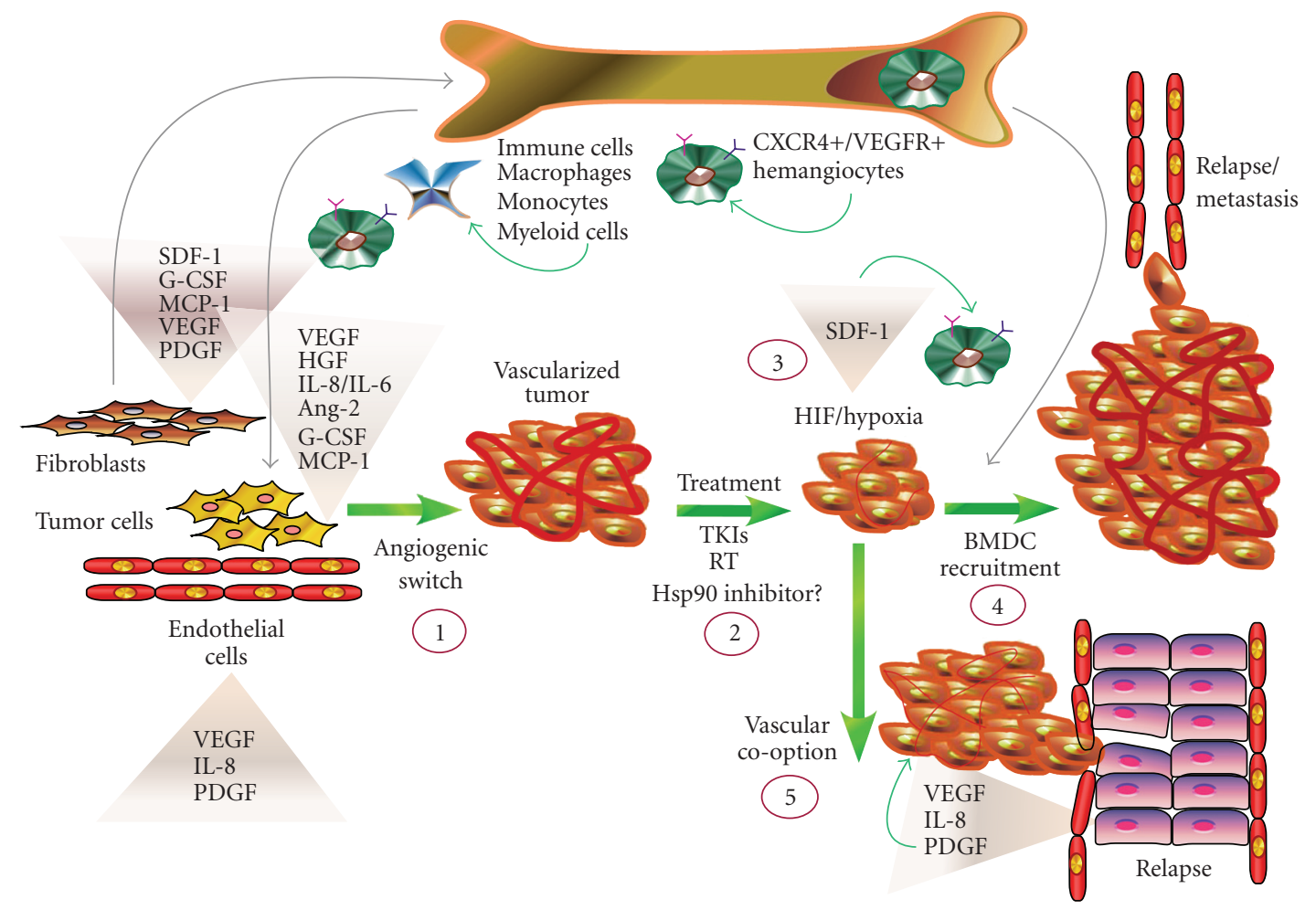

Figure 2: Potential for Hsp90 intervention strategies in curtailing angiogenic processes. Tumor stromal cells, such as cancer-associated fibroblasts (CAFs) and endothelial cells (ECs), communicate with tumor cells via their secretion of cytokines and thus contribute to the angiogenic switch (1). Cytokines from recruited BMDC progenitors contribute to this milieu to further stimulate tumor vascularization (1). Hsp90 inhibition may prevent HIF-driven cytokine release from tumor and stromal cells (i.e., SDF-1, VEGF, HGF, etc.), as well as HIF-mediated CXCR4+ expression in BMDCs, with the potential effect of curtailing recruitment of CXCR4+ progenitors to the tumor. Hsp90 inhibition also attenuates cytokine signaling via RTK inhibition (i.e., VEGFR, PDGFR), which may collectively prevent or delay the angiogenic switch. Therapeutic approaches utilizing radiotherapy (RT), tyrosine kinase inhibitors (TKIs), or Hsp90-targeted agents suppress tumor vascularization and growth (2). This initial reduction in vascularity may promote tumor hypoxia (3), subsequent HIF activation, and SDF-1 secretion, the latter of which may further stimulate BMDC recruitment (4). Hsp90 inhibitors are similarly predicted to suppress BMDC recruitment and HIF-driven cytokine secretion as in (1). Alternatively, when challenged with reduced HIF expression and decreased BMDC recruitment, tumor cells may coopt the vasculature of normal tissue (5). In this scenario, Hsp90 suppression is predicted to reduce the efficiency of EC-derived factors that support this process. The overall efficacy of Hsp90 inhibition upon tumor vascularization will depend upon the balance of Hsp90-dependent and Hsp90-independent signaling effectors driving the angiogenic process.

recognized as a risk factor for neoplastic transformation. This tumor supporting function is due to their ability to upregulate ECM proteins and secrete growth factors and cytokines, (i.e., fibroblast growth factor (FGF), connective tissue growth factor (CTGF), stromal-derived factor (SDF1) and VEGF), thereby creating conditions that constitute an optimal milieu for tumor development [151, 152]. CAFs robustly stimulate tumor angiogenesis and cancer progression in a number of xenograft models [153-157]. Myofibroblasts are also a component of nontumorigenic fibrotic tissue, such as in gastric, pancreatic, and hepatic stellate cells, which similarly secrete angiogenic cytokines and support paracrine signaling in adjacent epithelial cells [158]. Hypoxic signaling further drives fibrogenesis [159], and the hypoxia-mediated upregulation of VEGF and angiogenic cytokines from stellate cells perpetuates the fibrotic and hypoxic cycle $[158,160]$.

Given that myofibrolasts support and sustain cancer progression, there is heightened interest in agents that may target the CAF-rich stroma [161]. Although Hsp90targeted strategies are predicted to interfere with the VEGFR, PDGFR, and TGF beta receptor signaling that participates in tumor-stromal communication, reports examining the ability of either TKIs or Hsp90 inhibitors to attenuate $\mathrm{CAF} /$ stellate signaling are limited. Multi-TKIs reverse properties associated with the activated stellate phenotype in vitro and in vivo [162, 163], concomitant with reduced tumor vascularity [164], and Imatinib inhibits PDGF/AKT signaling and ECM production in breast stromal fibroblasts [165]. Hsp90 inhibitors exhibit cytotoxicity against CAFs derived from gastric cancer [44], which are potent mediators of tumor angiogenesis [166]. Interestingly, Hsp90 inhibition suppresses the reactive stromal phenotype in hepatic stellate cells, prior to initiating apoptosis [167], suggesting that Hsp90-targeted agents may be effective in reducing stromal support of tumorigenic progression.

Given that CAFs promote tumor vascularization in part via their ability to stimulate endothelial cell function, 
attention has now shifted to the ability of TKIs to cotarget the tumor endothelium. The stromal vasculature is comprised of endothelial cells and perivascular pericytes, the latter of which provide both survival signals and structural support to facilitate a mature and functional vasculature [168]. The pathways crucial to EC-pericyte communication and vascular stabilization include PDGF/PDGFR and VEGF/VEGFR signaling. Secretion of PDGF from cancer cells stimulates VEGF upregulation in pericytes via a PI3K/AKT mechanism, which protects ECs from apoptosis [169]. Furthermore, PDGF signaling is essential for tumor vascularization in a preclinical model of pancreatic cancer [170] and upregulated PDGF signaling is a characteristic of the tumor stroma in a preclinical model of cervical cancer [171]. In the latter example, expression of the PDGF receptor was primarily localized to stromal fibroblasts and pericytes, and inhibition of PDGFR signaling with Imatinib decreased pericyte coverage of tumor endothelial cells, concomitant with suppression of tumor vascularity and growth.

Hsp90 inhibitors are well characterized in their ability to suppress tumor vasculature and growth in multiple preclinical models; yet their specific effects upon endothelial cells and pericytes remain largely unexplored. A limited number of reports demonstrate that Hsp90 inhibition reduces proliferation, differentiation, motility, and angiogenic signaling in normal human endothelial cells $[72,139]$ and may elicit death of normal vascular smooth muscle cells (representative of pericytes) at high concentrations [44], but the molecular mechanisms for these effects remain obscure. The precise molecular effects of Hsp90 inhibition within the tumorassociated vasculature have not been clarified, and use of normal cells that resemble components of the tumor vasculature may not accurately reflect the tumor endothelium. Further, Hsp90 inhibitors may differentially destabilize proteins in tumor and vascular cells [101], illustrating the potential for differential tumor and stromal responses to therapeutics. Importantly, Hsp90 therapeutics may exhibit preferential uptake and selectivity for tumor tissue, proportional to the cancer specific hyperactivity of the Hsp90 chaperone $[172,173]$, inviting the question of whether these agents will effectively target components of the nontumorigenic stroma.

\subsection{The Tumor Stroma as an Enabler of Therapeutic Resis-} tance. Clinically, chemoresistance may be due to a failure to target the proangiogenic signaling derived from tumor stroma, a notion supported by the ability of CAFs to mediate drug sensitivity $[174,175]$. Endothelial cells also contribute to chemoresistance, as demonstrated by the use of temozolomide in the treatment glioblastoma multiforme (GBM). While temozolomide exhibits toxicity against GBM cells in culture, glioma-derived endothelial cells are refractory to treatment, thus offering a possible explanation for the clinical failure of these agents [176]. Similarly, the ability of pericytes to protect against EC apoptosis [169] implicates their potential role in attenuating the efficacy of chemotherapeutics. In support of this notion, dual inhibition of PDGFR and VEGFR signaling is synergistic in reducing pericyte coverage of tumor endothelium, and this combination is maximally effective in suppressing tumor growth over either agent alone in a mouse model of pancreatic islet cancer [177]. In addition to its role in chemoresistance, the tumor stroma also plays a major role in mediating the response to radiotherapy (RT). It is established that RT induces the expression and secretion of VEGF and proangiogenic cytokines in cancer cells, stromal endothelial cells, and fibroblasts [178-180]. This cytokine upregulation stimulated by the initiation of vascular repair mechanisms and HIF-dependent signaling $[181,182]$ represents a major barrier to chemosensitivity [183-185].

Antiangiogenic TKIs have met with limited success in combating radioresistance, despite their impairment of proangiogenic RTK signaling in both tumor and stromal cells [186]. Although not yet validated clinically, we, and others, have shown that Hsp90 inhibition imparts radiosensitivity to various cancer cell types in culture and in vivo [187-189]. While the basis for this enhancement is not well defined, Hsp90-dependent HIF targeting is one likely mechanism, supported by the finding that blockade of HIF/VEGF signaling, coupled with RT, potently destroys tumor vasculature in a xenograft model [190]. In further support of the ability of Hsp90 inhibition to impair this signaling axis, Hsp90directed therapy overrides the protective effects of VEGFmediated signaling in endothelial cells, in part through inhibition of AKT [191]. Since many of the RT-induced wound repair response pathways depend upon a number of Hsp90 clients, including HIF, AKT, MEK/ERK, PDGFR, and VEGFR, Hsp90 inhibition represents an alternate approach to impair multiple radioresponse regulatory proteins and improve outcome. In fact, it remains a possible, though unconfirmed notion, that the ability of Hsp90 inhibitors to destabilize interactions between ECs and pericytes may underlie their ability to suppress tumor vascularity and potentiate chemo- and radiosensitivity. However, given that optimal responses to the combination of anti-VEGF therapy with RT occur during the "normalization window" characterized by stabilization of the tumor vasculature subsequent to enhanced pericyte coverage [192], the use of Hsp90 inhibitors will have to be judiciously applied for optimal therapeutic benefit.

Similar to RT, vascular targeting agents exacerbate tumor hypoxia and activate HIF signaling, events antagonistic to treatment objectives. HIF activation stimulates cytokine secretion and recruitment of circulating BMDCs (Figure 2), which may replenish vascular components such as pericytes, CAFs, and myeloid cells. Mobilization of BMDCs to hypoxic areas promotes neovascularization and contributes to cancer aggressiveness and drug resistance [2, 193, 194]. Although factors regulating BMDC recruitment are not well defined, tumor hypoxia and the HIF-regulated cytokines VEGF and SDF-1 are established as major effectors in the recruitment of VEGFR and CXCR4 expressing BMDCs [49, 195, 196] (Figure 2). In a well-validated preclinical model, hypoxic or irradiated glioma cells each secreted factors that stimulated the homing of hematopoietic progenitors in a HIF-dependent manner [197]. The enhancement of tumor hypoxia via genetic or pharmacologic interference with VEGF signaling similarly recruited BMDCs and fueled aggressiveness [198, 199] (Figure 2). It remains to be seen 
whether Hsp90-directed therapy may effectively suppress HIF-mediated recruitment of BMDCs and whether attenuated recruitment may be sufficient for tumor suppression. Indeed, BMDC recruitment was diminished in a HIF-1 knockout mouse model of glioma; yet tumor cells became invasive and continued to thrive via cooption of the host vasculature [200]. In this example, the host cells were still competent to respond to tumor-mediated HIF/VEGF signaling, leaving open the possibility that Hsp90 inhibition, which would target HIF signaling in conjunction with VEGF mediated responses, may thwart this undesired adaptive event (Figure 2).

Another level of complexity is due to the nature of angiogenic compensation, in that HIF-independent compensatory mechanisms, such as $\mathrm{NF} \kappa \mathrm{B}$-dependent cytokine release, can also promote BMDC recruitment [49]. Hsp90 inhibitors have the potential to impair a subset of HIFindependent mechanisms, for example, by virtue of their ability to suppress effectors such as NF $\kappa$ B. Although the role of Hsp90-dependent signaling within the context of BMDCmediated neovascularization has not been explored, the ability of Hsp90 inhibitors to suppress BMDC stimulatory properties upon multiple myeloma cells in a coculture model [72] suggests that these agents may indeed have utility in preventing some aspects of BMDC-derived neovascularization. However, these agents are likely to incur a number limitations, given the recent identification of a subset of myeloid cells that are refractory to VEGF-dependent therapy, due to expression of G-CSF and Bv8, the latter of which drives local angiogenesis, as well as BMDC recruitment [199, 201]. As these ligands activate G-coupled protein receptors (GPCRs) that may not rely upon Hsp90 function, it is possible that this alternative angiogenic pathway will be immune to Hsp90-directed therapy. It therefore remains to be determined whether the lack of durable clinical response of Hsp90-directed therapy is due to an inability to suppress BMDC recruitment, to inhibit vascular cooption, or to suppress these compensatory angiogenic pathways. A further characterization of these pathways may pave the way to more successful regimens that employ rationally designed combination therapies.

4.5. The Road to Nirvana: Improving Preclinical Models, Drug Analyses, and Clinical Correlates. With the overarching goal of understanding the basis for clinical responses to Hsp90-targeted therapy, a continuum of models must be evaluated, each with its inherent strengths and weaknesses. Although cell-based in vitro models are necessary to decipher defined molecular signaling events, these systems do not recapitulate the complexity of the tumor microenvironment, such as dimensionality, matrix incorporation, or stromal communication, all factors that influence antiangiogenic responses [202, 203]. Xenograft models, which embody much of the complexity of the tumor microenvironment, are a necessary prerequisite for evaluating the utility of antiangiogenic agents in distinct cancers. However, the majority of these models utilize immunocompromised mice, which lack the full complement of immune components that may contribute to stromal dependent angiogenesis. Some of these differences invariably account for the ability of Hsp90 inhibitors, and other chemotherapeutics, to exhibit more potent tumor efficacy in preclinical models compared with their clinical responses. Immune competent genetic models of progressive cancer that more closely mimic the complexity of human malignancy represent valuable tools for dissection of stepwise genetic events in this process and may also help pinpoint when antiangiogenic agents would be most efficacious.

Another caveat to deciphering optimal treatment regimens is imparted by the molecular heterogeneity of cancers. Functionally, this is exemplified by the differential wiring of signaling networks, and the corresponding differential response to Hsp90 inhibition, a trait similarly reflected with other chemotherapeutics. These varying responses likely reflect the distinct molecular signatures driving cancer survival, each with a unique dependence upon Hsp90 function [204], combined with disparate local microenvironments from the periphery to the core. Moreover, differential client affinity in diverse cell and cancer types [205] hints at more complex molecular regulation. Further, the recent revelation that an average human tumor may have upwards of 20,000 mutations $[206,207]$ strongly supports the rationale for therapeutic strategies incorporating more broadly acting drugs, such as those represented by the category of Hsp90-directed agents. In addition to tumor cell heterogeneity, components of the tumor vasculature may demonstrate comparable complexity. In support of this notion, VEGF resistant CAFs may upregulate PDGF signaling [175] and promote chemoresistance. Similarly, the tumor endothelium exhibits significant heterogeneity of expressed surface molecules, as validated with use of phage display technology, based upon the principle of differential homing of peptides to the vasculature [208-210]. Interestingly, this approach identified distinct molecular signatures of the tumor endothelium that occur in a stage specific manner, with clinical implications for the variable tumor responses observed for TKIs. In a preclinical model of cervical cancer, a subset of TKI agents demonstrated preferential activity at distinct tumor stages, implicating their selective effects upon either tumor initiation or signaling events activated at later stages [171]. In this model, combination treatments (i.e., cotargeting VEGFR and PDGFR) demonstrated improved antitumor activity of progressive tumors, illuminating that optimal targeting approaches may need to consider prevention and intervention strategies as a component of the heterogeneity of the tumor-associated vasculature. Similar dynamic treatment analyses in well-defined preclinical models will further define optimal clinical usage for Hsp90 inhibitors.

In addition to allowing a dynamic evaluation of drug efficacy, preclinical models are useful tools to assess complex drug-dependent molecular changes. A subset of microRNAs was recently identified as components of the angiogenic switch in a genetic model of pancreatic cancer. Strikingly, these same targets were similarly upregulated in patients and modulated preclinically following treatment with Sunitinib [211]. Although a comparative detailed genetic analysis has not yet been performed with Hsp90 inhibitors, one group evaluated pharmacodynamic markers and resultant tumor 
angiogenesis [139], while another performed a proteomic and genetic analysis to identify a number of gene and protein changes [212]. Further preclinical models that incorporate dynamic molecular analyses have great potential for evaluating the effects of emerging Hsp90 inhibitors. Similarly, clinical trial design is likely to benefit from the incorporation of increasingly sophisticated molecular endpoints. Although the evaluation of changes in tumor burden and disease progression are established components of the clinical evaluation of anticancer agents, according to the RECIST (response evaluation criteria in solid tumors) criteria [213], these endpoints may not be optimal in obtaining nuanced information about tumor responses and changes within the tumor microenvironment. For example, in trials with Hsp90-targeted agents, a pertinent endpoint would entail the monitoring of drug-dependent depletion of relevant cancercausing client proteins. In a recent Phase II trial of 17-AAG in melanoma, differential drug responses were observed for client proteins from patient tumors [214], which may have implications for clinical outcome. Therefore, the ability to dynamically image Hsp90-dependent client depletion in vivo would be a clinically valuable addition to these trials. At least two reports demonstrate the feasibility of noninvasively viewing the dynamic expression of Hsp90 client proteins in real time in preclinical models $[215,216]$, suggesting that incorporation of these approaches into clinical trial design may be feasible.

More recently, a number of clinical trials have included increasingly sophisticated molecular endpoints to gauge drug responses. In a phase II trial of 17-AAG in metastatic prostate cancer, the relation between drug treatment and PSA was monitored, as were a limited number of serum derived cytokines and markers from peripheral blood [131]. In a carefully designed trial of a pan-VEGF inhibitor in GBM, MRI imaging of tumor vascularity discerned a window of vascular normalization [217]. Importantly, this group also identified several vascular modulators that correlated with tumor progression, such as circulating endothelial cells (CECs) and a subset of cytokines, such as SDF-1. In another detailed study by this group, a comprehensive genetic analysis of angiogenic markers revealed that antiVEGF therapy induced SDF-1 and inflammatory pathways in rectal cancer [218]. This integrative analysis of stage specific molecular and genetic alterations, coupled with the evaluation of surrogate biomarkers, represents a promising approach to better understand and possibly predict fluctuations in the clinical response to Hsp90 inhibitors. Looking forward, it must be determined whether and how Hsp90 inhibitors modulate signaling, angiogenic genes/microRNAs, cytokines, circulating endothelial and progenitor cells, tumor stroma, and which of these alterations are prognostic for tumor response or relapse. Whether the expanded repertoire of proteins targeted by Hsp90 inhibitors, compared with the relatively specific subset of proteins targeted by clinically approved antiangiogenic TKIs, will be an asset or a liability in the clinic remains to be determined. A more comprehensive understanding of the mode of action of Hsp90 inhibitors will position us to harness the power of these agents and design more effective strategies to diminish cancer lethality.

\section{Acknowledgments}

The authors wish to thank Linda Metheny-Barlow, Luke Whitesell, Bob Gemmill, and David Rowley for their support and critical review of this manuscript.

\section{References}

[1] J. Folkman, "Tumor angiogenesis: therapeutic implications," The New England Journal of Medicine, vol. 285, no. 21, pp. 1182-1186, 1971.

[2] M. Seandel, J. Butler, D. Lyden, and S. Raf, "A catalytic role for proangiogenic marrow-derived cells in tumor neovascularization," Cancer Cell, vol. 13, no. 3, pp. 181-183, 2008.

[3] G. Bergers and D. Hanahan, "Modes of resistance to antiangiogenic therapy," Nature Reviews Cancer, vol. 8, no. 8, pp. 592-603, 2008.

[4] S. Sanderson, M. Valenti, S. Gowan et al., "Benzoquinone ansamycin heat shock protein 90 inhibitors modulate multiple functions required for tumor angiogenesis," Molecular Cancer Therapeutics, vol. 5, no. 3, pp. 522-532, 2006.

[5] L. Whitesell, S. D. Shifrin, G. Schwab, and L. M. Neckers, "Benzoquinonoid ansamycins possess selective tumoricidal activity unrelated to src kinase inhibition," Cancer Research, vol. 52, no. 7, pp. 1721-1728, 1992.

[6] L. H. Pearl and C. Prodromou, "Structure and mechanism of the Hsp90 molecular chaperone machinery," Annual Review of Biochemistry, vol. 75, pp. 271-294, 2006.

[7] J. S. Isaacs, W. Xu, and L. Neckers, "Heat shock protein 90 as a molecular target for cancer therapeutics," Cancer Cell, vol. 3, no. 3, pp. 213-217, 2003.

[8] J. S. Isaacs, "Heat-shock protein 90 inhibitors in antineoplastic therapy: is it all wrapped up?" Expert Opinion on Investigational Drugs, vol. 14, no. 6, pp. 569-589, 2005.

[9] M. V. Powers and P. Workman, "Targeting of multiple signalling pathways by heat shock protein 90 molecular chaperone inhibitors," Endocrine-Related Cancer, vol. 13, supplement 1, pp. 125-135, 2006.

[10] L. Whitesell, R. Bagatell, and R. Falsey, "The stress response: implications for the clinical development of Hsp90 inhibitors," Current Cancer Drug Targets, vol. 3, no. 5, pp. 349-358, 2003.

[11] L. Neckers, "Hsp90 inhibitors as novel cancer chemotherapeutic agents," Trends in Molecular Medicine, vol. 8, no. 4, pp. S55-S61, 2002.

[12] S. M. Roe, C. Prodromou, R. O’Brien, J. E. Ladbury, P. W. Piper, and L. H. Pearl, "Structural basis for inhibition of the Hsp90 molecular chaperone by the antitumor antibiotics radicicol and geldanamycin," Journal of Medicinal Chemistry, vol. 42, no. 2, pp. 260-266, 1999.

[13] J. G. Supko, R. L. Hickman, M. R. Grever, and L. Malspeis, "Preclinical pharmacologic evaluation of geldanamycin as an antitumor agent," Cancer Chemotherapy and Pharmacology, vol. 36, no. 4, pp. 305-315, 1995.

[14] T. W. Schulte and L. M. Neckers, "The benzoquinone ansamycin 17-allylamino-17-demethoxygeldanamycin binds to HSP90 and shares important biologic activities with geldanamycin," Cancer Chemotherapy and Pharmacology, vol. 42, no. 4, pp. 273-279, 1998.

[15] Z.-Q. Tian, Y. Liu, D. Zhang et al., "Synthesis and biological activities of novel 17-aminogeldanamycin derivatives," Bioorganic and Medicinal Chemistry, vol. 12, no. 20, pp. 53175329, 2004. 
[16] S. Z. Usmani, R. Bona, and Z. Li, "17 AAG for HSP90 inhibition in cancer-from bench to bedside," Current Molecular Medicine, vol. 9, no. 5, pp. 654-664, 2009.

[17] H. M. Prince, M. J. Bishton, and R. W. Johnstone, "Panobinostat (LBH589): a potent pan-deacetylase inhibitor with promising activity against hematologic and solid tumors," Future Oncology, vol. 5, no. 5, pp. 601-612, 2009.

[18] Y. S. Kim, S. V. Alarcon, S. Lee et al., "Update on Hsp90 inhibitors in clinical trial," Current Topics in Medicinal Chemistry, vol. 9, no. 15, pp. 1479-1492, 2009.

[19] G. L. Semenza, "Defining the role of hypoxia-inducible factor 1 in cancer biology and therapeutics," Oncogene, vol. 29, no. 5, pp. 625-634, 2010.

[20] G. L. Semenza, "Involvement of hypoxia-inducible factor 1 in human cancer," Internal Medicine, vol. 41, no. 2, pp. 79-83, 2002.

[21] G. L. Semenza, "HIF-1, $\mathrm{O}_{2}$, and the 3 PHDs: how animal cells signal hypoxia to the nucleus," Cell, vol. 107, no. 1, pp. 1-3, 2001.

[22] M. Y. Koh and G. Powis, "HAF: the new player in oxygenindependent HIF- $1 \alpha$ degradation," Cell Cycle, vol. 8, no. 9, pp. 1359-1366, 2009.

[23] Y. S. Chun, M. S. Kim, and J. W. Park, "Oxygen-dependent and -independent regulation of HIF-1alpha," Journal of Korean Medical Science, vol. 17, no. 5, pp. 581-588, 2002.

[24] K. Kondo, J. Klco, E. Nakamura, M. Lechpammer, and W. G. Kaelin Jr., "Inhibition of HIF is necessary for tumor suppression by the von Hippel-Lindau protein," Cancer Cell, vol. 1, no. 3, pp. 237-246, 2002.

[25] W. G. Kaelin Jr., “The von Hippel-Lindau tumor suppressor protein and clear cell renal carcinoma," Clinical Cancer Research, vol. 13, no. 2, pp. 680S-684S, 2007.

[26] E. B. Rankin and A. J. Giaccia, "The role of hypoxia-inducible factors in tumorigenesis," Cell Death and Differentiation, vol. 15, no. 4, pp. 678-685, 2008.

[27] O. Iliopoulos, A. P. Levy, C. Jiang, W. G. Kaelin Jr., and M. A. Goldberg, "Negative regulation of hypoxia-inducible genes by the von Hippel-Lindau protein," Proceedings of the National Academy of Sciences of the United States of America, vol. 93, no. 20, pp. 10595-10599, 1996.

[28] D. Liao and R. S. Johnson, "Hypoxia: a key regulator of angiogenesis in cancer," Cancer and Metastasis Reviews, vol. 26, no. 2, pp. 281-290, 2007.

[29] W. Wang, W.-D. Jia, G.-L. Xu et al., "Antitumoral activity of rapamycin mediated through inhibition of HIF-1alpha and VEGF in hepatocellular carcinoma," Digestive Diseases and Sciences, vol. 54, no. 10, pp. 2128-2136, 2009.

[30] D. Del Bufalo, L. Ciuffreda, D. Trisciuoglio et al., "Antiangiogenic potential of the mammalian target of rapamycin inhibitor temsirolimus," Cancer Research, vol. 66, no. 11, pp. 5549-5554, 2006.

[31] M. Puppo, F. Battaglia, C. Ottaviano et al., "Topotecan inhibits vascular endothelial growth factor production and angiogenic activity induced by hypoxia in human neuroblastoma by targeting hypoxia-inducible factor- $1 \alpha$ and $-2 \alpha$," Molecular Cancer Therapeutics, vol. 7, no. 7, pp. 1974-1984, 2008.

[32] A. Rapisarda, B. Uranchimeg, O. Sordet, Y. Pommier, R. H. Shoemaker, and G. Melillo, "Topoisomerase I-mediated inhibition of hypoxia-inducible factor 1: mechanism and therapeutic implications," Cancer Research, vol. 64, no. 4, pp. 1475-1482, 2004.
[33] M. Y. Koh, T. Spivak-Kroizman, S. Venturini et al., "Molecular mechanisms for the activity of PX-478, an antitumor inhibitor of the hypoxia-inducible factor- $1 \alpha$," Molecular Cancer Therapeutics, vol. 7, no. 1, pp. 90-100, 2008.

[34] L. M. Greenberger, I. D. Horak, D. Filpula et al., "A RNA antagonist of hypoxia-inducible factor-1 $\alpha$, EZN-2968, inhibits tumor cell growth," Molecular Cancer Therapeutics, vol. 7, no. 11, pp. 3598-3608, 2008.

[35] E. Minet, D. Mottet, G. Michel et al., "Hypoxia-induced activation of HIF-1: role of HIF- $1 \alpha$-Hsp 90 interaction," FEBS Letters, vol. 460, no. 2, pp. 251-256, 1999.

[36] D. M. Katschinski, L. Le, S. G. Schindler, T. Thomas, A. K. Voss, and R. H. Wenger, "Interaction of the PAS B domain with HSP90 accelerates hypoxia-inducible factor- $1 \alpha$ stabilization," Cellular Physiology and Biochemistry, vol. 14, no. 4-6, pp. 351-360, 2004.

[37] J. S. Isaacs, Y. J. Jung, E. G. Mimnaugh, A. Martinez, F. Cuttitta, and L. M. Neckers, "Hsp90 regulates a von Hippel Lindau-independent hypoxia-inducible factor-1 alphadegradative pathway," Journal of Biological Chemistry, vol. 277, no. 33, pp. 29936-29944, 2002.

[38] J. Yang, L. Zhang, P. J. A. Erbel et al., "Functions of the Per/ARNT/Sim domains of the hypoxia-inducible factor," Journal of Biological Chemistry, vol. 280, no. 43, pp. 3604736054, 2005.

[39] M. Milkiewicz, J. L. Doyle, T. Fudalewski, E. Ispanovic, M. Aghasi, and T. L. Haas, "HIF- $1 \alpha$ and HIF- $2 \alpha$ play a central role in stretch-induced but not shear-stress-induced angiogenesis in rat skeletal muscle," Journal of Physiology, vol. 583, no. 2, pp. 753-766, 2007.

[40] J. S. Isaacs, Y.-J. Jung, and L. Neckers, "Aryl hydrocarbon nuclear translocator (ARNT) promotes oxygen-independent stabilization of hypoxia-inducible factor- $1 \alpha$ by modulating an Hsp90-dependent regulatory pathway," Journal of Biological Chemistry, vol. 279, no. 16, pp. 16128-16135, 2004.

[41] Y. V. Liu, J. H. Baek, H. Zhang, R. Diez, R. N. Cole, and G. L. Semenza, "RACK1 competes with HSP90 for binding to HIF$1 \alpha$ and is required for O2-independent and HSP90 inhibitorinduced degradation of HIF-1 $\alpha$," Molecular Cell, vol. 25, no. 2, pp. 207-217, 2007.

[42] S. Amir, R. Wang, J. W. Simons, and N. J. Mabjeesh, "SEPT9_v1 up-regulates hypoxia-inducible factor 1 by preventing its RACK1-mediated degradation," Journal of Biological Chemistry, vol. 284, no. 17, pp. 11142-11151, 2009.

[43] O. Alqawi, M. Moghaddas, and G. Singh, "Effects of geldanamycin on HIF- $1 \alpha$ mediated angiogenesis and invasion in prostate cancer cells," Prostate Cancer and Prostatic Diseases, vol. 9, no. 2, pp. 126-135, 2006.

[44] S. A. Lang, D. Klein, C. Moser et al., "Inhibition of heat shock protein 90 impairs epidermal growth factor-mediated signaling in gastric cancer cells and reduces tumor growth and vascularization in vivo," Molecular Cancer Therapeutics, vol. 6, no. 3, pp. 1123-1132, 2007.

[45] S. A. Lang, C. Moser, A. Gaumann et al., "Targeting heat shock protein 90 in pancreatic cancer impairs insulin-like growth factor-I receptor signaling, disrupts an interleukin6/signal-transducer and activator of transcription 3/hypoxiainducible factor- $1 \alpha$ autocrine loop, and reduces orthotopic tumor growth," Clinical Cancer Research, vol. 13, no. 21, pp. 6459-6468, 2007.

[46] Y. Mizukami, Y. Kohgo, and D. C. Chung, "Hypoxia inducible factor-1-independent pathways in tumor angiogenesis," Clinical Cancer Research, vol. 13, no. 19, pp. 5670-5674, 2007. 
[47] D. Martin, R. Galisteo, and Gutkind J. S., "CXCL8/IL8 stimulates vascular endothelial growth factor (VEGF) expression and the autocrine activation of VEGFR2 in endothelial cells by activating NFkappaB through the CBM (Carma3/Bcl10/Malt1) complex," The Journal of Biological Chemistry, vol. 284, no. 10, pp. 6038-6042, 2009.

[48] D. J. Brat, A. C. Bellail, and E. G. Van Meir, "The role of interleukin-8 and its receptors in gliomagenesis and tumoral angiogenesis," Neuro-Oncology, vol. 7, no. 2, pp. 122-133, 2005.

[49] D. A. Chan, T. L.A. Kawahara, P. D. Sutphin, H. Y. Chang, J.T. Chi, and A. J. Giaccia, "Tumor vasculature is regulated by PHD2-mediated angiogenesis and bone marrow-derived cell recruitment," Cancer Cell, vol. 15, no. 6, pp. 527-538, 2009.

[50] S. Huang, J. B. Robinson, A. DeGuzman, C. D. Bucana, and I. J. Fidler, "Blockade of nuclear factor- $\kappa$ b signaling inhibits angiogenesis and tumorigenicity of human ovarian cancer cells by suppressing expression of vascular endothelial growth factor and interleukin 8," Cancer Research, vol. 60, no. 19, pp. 5334-5339, 2000.

[51] J. Rius, M. Guma, C. Schachtrup et al., "NF- $\kappa$ B links innate immunity to the hypoxic response through transcriptional regulation of HIF-1 $\alpha$," Nature, vol. 453, no. 7196, pp. 807$811,2008$.

[52] M. Broemer, D. Krappmann, and C. Scheidereit, "Requirement of Hsp90 activity for I $\kappa \mathrm{B}$ kinase (IKK) biosynthesis and for constitutive and inducible IKK and NF- $\kappa \mathrm{B}$ activation," Oncogene, vol. 23, no. 31, pp. 5378-5386, 2004.

[53] J. Crèvecoeur, M.-P. Merville, J. Piette, and G. Gloire, "Geldanamycin inhibits tyrosine phosphorylation-dependent NF- $\kappa$ B activation," Biochemical Pharmacology, vol. 75, no. 11, pp. 2183-2191, 2008.

[54] G. Qing, P. Yan, Z. Qu, H. Liu, and G. Xiao, "Hsp90 regulates processing of $\mathrm{NF}-\kappa \mathrm{B} 2$ p100 involving protection of NF- $\kappa \mathrm{B}$-inducing kinase (NIK) from autophagy-mediated degradation," Cell Research, vol. 17, no. 6, pp. 520-530, 2007.

[55] J. Lewis, A. Devin, A. Miller et al., "Disruption of Hsp96 function results in degradation of the death domain kinase, receptor-interacting protein (RIP), and blockage of tumor necrosis factor-induced nuclear factor- $\kappa \mathrm{B}$ activation," Journal of Biological Chemistry, vol. 275, no. 14, pp. 10519-10526, 2000.

[56] M. Ammirante, A. Rosati, A. Gentilella et al., "The activity of hsp $90 \alpha$ promoter is regulated by NF- $\kappa \mathrm{B}$ transcription factors," Oncogene, vol. 27, no. 8, pp. 1175-1178, 2008.

[57] A. Papapetropoulos, G. García-Cardeña, J. A. Madri, and W. C. Sessa, "Nitric oxide production contributes to the angiogenic properties of vascular endothelial growth factor in human endothelial cells," Journal of Clinical Investigation, vol. 100, no. 12, pp. 3131-3139, 1997.

[58] J. P. Cooke, "NO and angiogenesis," Atherosclerosis Supplements, vol. 4, no. 4, pp. 53-60, 2003.

[59] G. Van Buren II, E. R. Camp, A. D. Yang et al., "The role of nitric oxide in mediating tumour blood flow," Expert Opinion on Therapeutic Targets, vol. 10, no. 5, pp. 689-701, 2006.

[60] M. Duval, F. Le Bœuf, J. Huot, and J.-P. Gratton, "Srcmediated phosphorylation of Hsp90 in response to vascular endothelial growth factor (VEGF) is required for VEGF receptor-2 signaling to endothelial NO synthase," Molecular Biology of the Cell, vol. 18, no. 11, pp. 4659-4668, 2007.

[61] J. Fontana, D. Fulton, Y. Chen et al., "Domain mapping studies reveal that the $\mathrm{M}$ domain of hsp90 serves as a molecular scaffold to regulate Akt-dependent phosphorylation of endothelial nitric oxide synthase and NO release," Circulation Research, vol. 90, no. 8, pp. 866-873, 2002.

[62] J.-X. Chen and B. Meyrick, "Hypoxia increases Hsp90 binding to eNOS via PI3K-Akt in porcine coronary artery endothelium," Laboratory Investigation, vol. 84, no. 2, pp. 182-190, 2004.

[63] F. Roviezzo, M. Bucci, C. Delisle et al., "Essential requirement for sphingosine kinase activity in eNOS-dependent $\mathrm{NO}$ release and vasorelaxation," FASEB Journal, vol. 20, no. 2, pp. 340-342, 2006.

[64] V. Anelli, C. R. Gault, A. B. Cheng, and L. M. Obeid, "Sphingosine kinase 1 is up-regulated during hypoxia in U87MG glioma cells: Role of hypoxia-inducible factors 1 and 2," Journal of Biological Chemistry, vol. 283, no. 6, pp. 33653375, 2008.

[65] M. D. Michaud, G. A. Robitaille, J.-P. Gratton, and D. E. Richard, "Sphingosine-1-phosphate: a novel nonhypoxic activator of hypoxia-inducible factor-1 in vascular cells," Arteriosclerosis, Thrombosis, and Vascular Biology, vol. 29, no. 6, pp. 902-908, 2009.

[66] S. E. Schnitzer, A. Weigert, J. Zhou, and B. Brüne, "Hypoxia enhances sphingosine kinase 2 activity and provokes sphingosine-1-phosphate-mediated chemoresistance in A549 lung cancer cells," Molecular Cancer Research, vol. 7, no. 3, pp. 393-401, 2009.

[67] S. Sato, N. Fujita, and T. Tsuruo, "Modulation of Akt kinase activity by binding to Hsp90," Proceedings of the National Academy of Sciences of the United States of America, vol. 97, no. 20, pp. 10832-10837, 2000.

[68] A. Citri, D. Harari, G. Shohat et al., "Hsp90 recognizes a common surface on client kinases," Journal of Biological Chemistry, vol. 281, no. 20, pp. 14361-14369, 2006.

[69] K. A. Pritchard Jr., A. W. Ackerman, E. R. Gross et al., "Heat shock protein 90 mediates the balance of nitric oxide and superoxide anion from endothelial nitric-oxide synthase," Journal of Biological Chemistry, vol. 276, no. 21, pp. 1762117624, 2001.

[70] G. García-Cardeña, R. Fan, V. Shah et al., "Dynamic activation of endothelial nitric oxide synthase by Hsp90," Nature, vol. 392, no. 6678, pp. 821-824, 1998.

[71] J. Sun and J. K. Liao, "Induction of angiogenesis by heat shock protein 90 mediated by protein kinase Akt and endothelial nitric oxide synthase," Arteriosclerosis, Thrombosis, and Vascular Biology, vol. 24, no. 12, pp. 2238-2244, 2004.

[72] Y. Okawa, T. Hideshima, P. Steed et al., "SNX-2112, a selective Hsp90 inhibitor, potently inhibits tumor cell growth, angiogenesis, and osteoclastogenesis in multiple myeloma and other hematologic tumors by abrogating signaling via Akt and ERK," Blood, vol. 113, no. 4, pp. 846-855, 2009.

[73] J. Y. Hsu and H. A. Wakelee, "Monoclonal antibodies targeting vascular endothelial growth factor: current status and future challenges in cancer therapy," BioDrugs, vol. 23, no. 5, pp. 289-304, 2009.

[74] L. M. Ellis and D. J. Hicklin, "VEGF-targeted therapy: mechanisms of anti-tumour activity," Nature Reviews Cancer, vol. 8, no. 8, pp. 579-591, 2008.

[75] M. C. Heinrich, C. L. Corless, G. D. Demetri et al., "Kinase mutations and imatinib response in patients with metastatic gastrointestinal stromal tumor," Journal of Clinical Oncology, vol. 21, no. 23, pp. 4342-4349, 2003.

[76] J. A. Engelman and J. Settleman, "Acquired resistance to tyrosine kinase inhibitors during cancer therapy," Current Opinion in Genetics and Development, vol. 18, no. 1, pp. 7379, 2008. 
[77] S. Yano, W. Wang, Q. Li et al., "Hepatocyte growth factor induces gefitinib resistance of lung adenocarcinoma with epidermal growth factor receptor-activating mutations," Cancer Research, vol. 68, no. 22, pp. 9479-9487, 2008.

[78] L. Ellis and D. J. Hicklin, "Pathways mediating resistance to vascular endothelial growth factor-targeted therapy," Clinical Cancer Research, vol. 14, no. 20, pp. 6371-6375, 2008.

[79] J. M. Stommel, A. C. Kimmelman, H. Ying et al., "Coactivation of receptor tyrosine kinases affects the response of tumor cells to targeted therapies," Science, vol. 318, no. 5848, pp. 287-290, 2007.

[80] L. Whitesell and S. L. Lindquist, "HSP90 and the chaperoning of cancer," Nature Reviews Cancer, vol. 5, no. 10, pp. 761-772, 2005.

[81] D. George, "Platelet-derived growth factor receptors: a therapeutic target in solid tumors," Seminars in Oncology, vol. 28, no. 5, pp. 27-33, 2001.

[82] D. Wang, H.-J. S. Huang, A. Kazlauskas, and W. K. Cavenee, "Induction of vascular endothelial growth factor expression in endothelial cells by platelet-derived growth factor through the activation of phosphatidylinositol 3-kinase," Cancer Research, vol. 59, no. 7, pp. 1464-1472, 1999.

[83] L. J. Nissen, R. Cao, E.-M. Hedlund et al., "Angiogenic factors FGF2 and PDGF-BB synergistically promote murine tumor neovascularization and metastasis," Journal of Clinical Investigation, vol. 117, no. 10, pp. 2766-2777, 2007.

[84] R. Cao, A. Eriksson, H. Kubo, K. Alitalo, Y. Cao, and J. Thyberg, "Comparative evaluation of FGF-2-, VEGF-A-, and VEGF-C-induced angiogenesis lymphangiogenesis, vascular fenestrations, and permeability," Circulation Research, vol. 94, no. 5, pp. 664-670, 2004.

[85] P. A. Jänne, N. Gray, and J. Settleman, "Factors underlying sensitivity of cancers to small-molecule kinase inhibitors," Nature Reviews Drug Discovery, vol. 8, no. 9, pp. 709-723, 2009.

[86] D. J. George, "Phase 2 studies of sunitinib and AG013736 in patients with cytokine-refractory renal cell carcinoma," Clinical Cancer Research, vol. 13, no. 2, part 2, pp. 753-757, 2007.

[87] S. Pennacchietti, P. Michieli, M. Galluzzo, M. Mazzone, S. Giordano, and P. M. Comoglio, "Hypoxia promotes invasive growth by transcriptional activation of the met protooncogene," Cancer Cell, vol. 3, no. 4, pp. 347-361, 2003.

[88] J. Wojta, C. Kaun, J. M. Breuss et al., "Hepatocyte growth factor increases expression of vascular endothelial growth factor and plasminogen activator inhibitor-1 in human keratinocytes and the vascular endothelial growth factor receptor flk-1 in human endothelial cells," Laboratory Investigation, vol. 79, no. 4, pp. 427-438, 1999.

[89] F. Koga, S. Tsutsumi, and L. M. Neckers, "Low dose geldanamycin inhibits hepatocyte growth factor and hypoxiastimulated invasion of cancer cells," Cell Cycle, vol. 6, no. 11, pp. 1393-1402, 2007.

[90] A. M. Petit, J. Rak, M.-C. Hung et al., "Neutralizing antibodies against epidermal growth factor and ErbB-2/neu receptor tyrosine kinases down-regulate vascular endothelial growth factor production by tumor cells in vitro and in vivo: angiogenic implications for signal transduction therapy of solid tumors," American Journal of Pathology, vol. 151, no. 6, pp. 1523-1530, 1997.

[91] Y. M. Lee, M. H. Bae, O. H. Lee et al., "Synergistic induction of in vivo angiogenesis by the combination of insulin-like growth factor-II and epidermal growth factor," Oncology Reports, vol. 12, no. 4, pp. 843-848, 2004.
[92] S. Wang, I. Pashtan, S. Tsutsumi, W. Xu, and L. Neckers, "Cancer cells harboring MET gene amplification activate alternative signaling pathways to escape MET inhibition but remain sensitive to Hsp90 inhibitors," Cell Cycle, vol. 8, no. 13, pp. 2050-2056, 2009.

[93] R. Sordella, D. W. Bell, D. A. Haber, and J. Settleman, "Gefitinib-sensitizing EGFR mutations in lung cancer activate anti-apoptotic pathways," Science, vol. 305, no. 5687, pp. 1163-1167, 2004.

[94] J. G. Paez, P. A. Jänne, J. C. Lee et al., "EGFR mutations in lung, cancer: Correlation with clinical response to gefitinib therapy," Science, vol. 304, no. 5676, pp. 1497-1500, 2004.

[95] E. R. Camp, J. Summy, T. W. Bauer, W. Liu, G. E. Gallick, and L. M. Ellis, "Molecular mechanisms of resistance to therapies targeting the epidermal growth factor receptor," Clinical Cancer Research, vol. 11, no. 1, pp. 397-405, 2005.

[96] I. R. Hutcheson, J. M. Knowlden, H. E. Jones et al., "Inductive mechanisms limiting response to anti-epidermal growth factor receptor therapy," Endocrine-Related Cancer, vol. 13, supplement 1, pp. 89-97, 2006.

[97] M. Guix, A. C. Faber, S. E. Wang et al., "Acquired resistance to EGFR tyrosine kinase inhibitors in cancer cells is mediated by loss of IGF-binding proteins," Journal of Clinical Investigation, vol. 118, no. 7, pp. 2609-2619, 2008.

[98] A. C. Faber, D. Li, Y. Song et al., "Differential induction of apoptosis in HER2 and EGFR addicted cancers following PI3K inhibition," Proceedings of the National Academy of Sciences of the United States of America, vol. 106, no. 46, pp. 19503-19508, 2010.

[99] C. Moser, P. Schachtschneider, S. A. Lang et al., "Inhibition of insulin-like growth factor-I receptor (IGF-IR) using NVPAEW541, a small molecule kinase inhibitor, reduces orthotopic pancreatic cancer growth and angiogenesis," European Journal of Cancer, vol. 44, no. 11, pp. 1577-1586, 2008.

[100] C. P. Webb, C. D. Hose, S. Koochekpour et al., "The geldanamycins are potent inhibitors of the hepatocyte growth factor/scatter factor-met-urokinase plasminogen activatorplasmin proteolytic network," Cancer Research, vol. 60, no. 2, pp. 342-349, 2000.

[101] D. Matei, M. Satpathy, L. Cao, Y.-C. Lai, H. Nakshatri, and D. B. Donner, "The platelet-derived growth factor receptor $\alpha$ is destabilized by geldanamycins in cancer cells," Journal of Biological Chemistry, vol. 282, no. 1, pp. 445-453, 2007.

[102] F. F. Zheng, S. D. Kuduk, G. Chiosis et al., "Identification of a geldanamycin dimer that induces the selective degradation of HER-family tyrosine kinases," Cancer Research, vol. 60, no. 8, pp. 2090-2094, 2000.

[103] S. J. Lavictoire, D. A. E. Parolin, A. C. Klimowicz, J. F. Kelly, and I. A. J. Lorimer, "Interaction of Hsp90 with the nascent form of the mutant epidermal growth factor receptor EGFRvIII," Journal of Biological Chemistry, vol. 278, no. 7, pp. 5292-5299, 2003.

[104] T. Shimamura, A. M. Lowell, J. A. Engelman, and G. I. Shapiro, "Epidermal growth factor receptors harboring kinase domain mutations associate with the heat shock protein 90 chaperone and are destabilized following exposure to geldanamycins," Cancer Research, vol. 65, no. 14, pp. 64016408, 2005.

[105] S. Modi, A. T. Stopeck, M. S. Gordon et al., "Combination of trastuzumab and tanespimycin (17-AAG, KOS-953) is safe and active in trastuzumab-refractory HER-2-overexpressing breast cancer: a phase I dose-escalation study," Journal of Clinical Oncology, vol. 25, no. 34, pp. 5410-5417, 2007. 
[106] H.-J. Ochel, T. W. Schulte, P. Nguyen, J. Trepel, and L. Neckers, "The benzoquinone ansamycin geldanamycin stimulates proteolytic degradation of focal adhesion kinase," Molecular Genetics and Metabolism, vol. 66, no. 1, pp. 24-30, 1999.

[107] T. W. Schulte, M. V. Blagosklonny, L. Romanova et al., "Destabilization of Raf-1 by geldanamycin leads to disruption of the Raf- 1-MEK-mitogen-activated protein kinase signalling pathway," Molecular and Cellular Biology, vol. 16, no. 10 , pp. 5839-5845, 1996.

[108] D. B. Solit, A. D. Basso, A. B. Olshen, H. I. Scher, and N. Rosen, "Inhibition of heat shock protein 90 function downregulates Akt kinase and sensitizes tumors to Taxol," Cancer Research, vol. 63, no. 9, pp. 2139-2144, 2003.

[109] L. K. Francis, Y. Alsayed, X. Leleu et al., "Combination mammalian target of rapamycin inhibitor rapamycin and HSP90 inhibitor 17-allylamino-17-demethoxygeldanamycin has synergistic activity in multiple myeloma," Clinical Cancer Research, vol. 12, no. 22, pp. 6826-6835, 2006.

[110] D. R. Premkumar, B. Arnold, and I. F. Pollack, "Cooperative inhibitory effect of ZD1839 (Iressa) in combination with 17AAG on glioma cell growth," Molecular Carcinogenesis, vol. 45, no. 5, pp. 288-301, 2006.

[111] M. A. Park, G. Zhang, C. Mitchell et al., "Mitogen-activated protein kinase kinase $1 / 2$ inhibitors and 17-allylamino-17demethoxygeldanamycin synergize to kill human gastrointestinal tumor cells in vitro via suppression of c-FLIP-s levels and activation of CD95," Molecular Cancer Therapeutics, vol. 7, no. 9, pp. 2633-2648, 2008.

[112] J. S. Abramson, W. Chen, P. Juszczynski et al., "The heat shock protein 90 inhibitor IPI-504 induces apoptosis of AKTdependent diffuse large B-cell lymphomas," British Journal of Haematology, vol. 144, no. 3, pp. 358-366, 2009.

[113] E. B. Pasquale, "Eph-ephrin bidirectional signaling in physiology and disease," Cell, vol. 133, no. 1, pp. 38-52, 2008.

[114] D. M. Brantley-Sieders, J. Caughron, D. Hicks, A. Pozzi, J. C. Ruiz, and J. Chen, "EphA2 receptor tyrosine kinase regulates endothelial cell migration and vascular assembly through phosphoinositide 3-kinase-mediated Rac1 GTPase activation," Journal of Cell Science, vol. 117, no. 10, pp. 20372049, 2004.

[115] D. M. Brantley-Sieders, G. Zhuang, D. Hicks et al., "The receptor tyrosine kinase EphA2 promotes mammary adenocarcinoma tumorigenesis and metastatic progression in mice by amplifying ErbB2 signaling," Journal of Clinical Investigation, vol. 118, no. 1, pp. 64-78, 2008.

[116] D. M. Brantley-Sieders and J. Chen, "Eph receptor tyrosine kinases in angiogenesis: from development to disease," Angiogenesis, vol. 7, no. 1, pp. 17-28, 2004.

[117] M. Héroult, F. Schaffner, and H. G. Augustin, "Eph receptor and ephrin ligand-mediated interactions during angiogenesis and tumor progression," Experimental Cell Research, vol. 312, no. 5, pp. 642-650, 2006.

[118] N. K. Noren and E. B. Pasquale, "Paradoxes of the EphB4 receptor in cancer," Cancer Research, vol. 67, no. 9, pp. 39943997, 2007.

[119] J. Wykosky, D. M. Gibo, C. Stanton, and W. Debinski, "EphA2 as a novel molecular marker and target in glioblastoma multiforme," Molecular Cancer Research, vol. 3, no. 10, pp. 541-551, 2005.

[120] H. Miao, D.-Q. Li, A. Mukherjee et al., "EphA2 mediates ligand-dependent inhibition and ligand-independent promotion of cell migration and invasion via a reciprocal regulatory loop with Akt," Cancer Cell, vol. 16, no. 1, pp. 920, 2009.

[121] C. N. Landen Jr., A. Chavez-Reyes, C. Bucana et al., "Therapeutic EphA2 gene targeting in vivo using neutral liposomal small interfering RNA delivery," Cancer Research, vol. 65, no. 15, pp. 6910-6918, 2005.

[122] C. N. Landen, C. Lu, L. Y. Han et al., "Efficacy and antivascular effects of EphA2 reduction with an agonistic antibody in ovarian cancer," Journal of the National Cancer Institute, vol. 98, no. 21, pp. 1558-1570, 2006.

[123] B. Annamalai, X. Liu, U. Gopal, and J. S. Isaacs, "Hsp90 is an essential regulator of EphA2 receptor stability and signaling: implications for cancer cell migration and metastasis," Molecular Cancer Research, vol. 7, no. 7, pp. 1021-1032, 2009.

[124] M. Macrae, R. M. Neve, P. Rodriguez-Viciana et al., "A conditional feedback loop regulates Ras activity through EphA2," Cancer Cell, vol. 8, no. 2, pp. 111-118, 2005.

[125] A. B. Larsen, M. W. Pedersen, M.-T. Stockhausen, M. V. Grandal, B. Van Deurs, and H. S. Poulsen, "Activation of the EGFR gene target EphA2 inhibits epidermal growth factorinduced cancer cell motility," Molecular Cancer Research, vol. 5, no. 3, pp. 283-293, 2007.

[126] D. M. Brantley-Sieders, W. B. Fang, Y. Hwang, D. Hicks, and J. Chen, "Ephrin-A1 facilitates mammary tumor metastasis through an angiogenesis-dependent mechanism mediated by EphA receptor and vascular endothelial growth factor in mice," Cancer Research, vol. 66, no. 21, pp. 10315-10324, 2006.

[127] Y. R. Chin and A. Toker, "Function of Akt/PKB signaling to cell motility, invasion and the tumor stroma in cancer," Cellular Signalling, vol. 21, no. 4, pp. 470-476, 2009.

[128] S. Chandarlapaty, A. Sawai, Q. Ye et al., "SNX2112, a synthetic heat shock protein 90 inhibitor, has potent antitumor activity against HER kinase-dependent cancers," Clinical Cancer Research, vol. 14, no. 1, pp. 240-248, 2008.

[129] A. Franovic, C. E. Holterman, J. Payette, and S. Lee, "Human cancers converge at the HIF- $2 \alpha$ oncogenic axis," Proceedings of the National Academy of Sciences of the United States of America, vol. 106, no. 50, pp. 21306-21311, 2009.

[130] K. Burkitt, S. Y. Chun, D. T. Dang, and L. H. Dang, "Targeting both HIF-1 and HIF-2 in human colon cancer cells improves tumor response to sunitinib treatment," Molecular Cancer Therapeutics, vol. 8, no. 5, pp. 1148-1156, 2009.

[131] E. I. Heath, D. W. Hillman, U. Vaishampayan et al., "A phase II trial of 17-allylamino-17-demethoxygeldanamycin in patients with hormone-refractory metastatic prostate cancer," Clinical Cancer Research, vol. 14, no. 23, pp. 79407946, 2008.

[132] E. A. Ronnen, G. V. Kondagunta, N. Ishill et al., "A phase II trial of 17-(Allylamino)-17-demethoxygeldanamycin in patients with papillary and clear cell renal cell carcinoma," Investigational New Drugs, vol. 24, no. 6, pp. 543-546, 2006.

[133] L. R. Kelland, S. Y. Sharp, P. M. Rogers, T. G. Myers, and P. Workman, "DT-diaphorase expression and tumor cell sensitivity to 17-allylamino,17- demethoxygeldanamycin, an inhibitor of heat shock protein 90," Journal of the National Cancer Institute, vol. 91, no. 22, pp. 1940-1949, 1999.

[134] N. Gaspar, S. Y. Sharp, S. Pacey et al., "Acquired resistance to 17-allylamino-17-demethoxygeldanamycin (17-AAG, tanespimycin) in glioblastoma cells," Cancer Research, vol. 69, no. 5, pp. 1966-1975, 2009. 
[135] W. Guo, P. Reigan, D. Siegel, J. Zirrolli, D. Gustafson, and D. Ross, "Formation of 17-allylamino-demethoxygeldanamycin (17-AAG) hydroquinone by $\mathrm{NAD}(\mathrm{P}) \mathrm{H}$ :quinone oxidoreductase 1: role of 17-AAG hydroquinone in heat shock protein 90 inhibition," Cancer Research, vol. 65, no. 21, pp. 1000610015, 2005.

[136] W. Guo, P. Reigan, D. Siegel, and D. Ross, "Enzymatic reduction and glutathione conjugation of benzoquinone ansamycin heat shock protein 90 inhibitors: relevance for toxicity and mechanism of action," Drug Metabolism and Disposition, vol. 36, no. 10, pp. 2050-2057, 2008.

[137] G. Chiosis, B. Lucas, A. Shtil, H. Huezo, and N. Rosen, "Development of a purine-scaffold novel class of hsp90 binders that inhibit the proliferation of cancer cells and induce the degradation of Her2 tyrosine kinase," Bioorganic and Medicinal Chemistry, vol. 10, no. 11, pp. 3555-3564, 2002.

[138] D. B. Solit and G. Chiosis, "Development and application of Hsp90 inhibitors," Drug Discovery Today, vol. 13, no. 1-2, pp. 38-43, 2008.

[139] S. A. Eccles, A. Massey, F. I. Raynaud et al., "NVP-AUY922: a novel heat shock protein 90 inhibitor active against xenograft tumor growth, angiogenesis, and metastasis," Cancer Research, vol. 68, no. 8, pp. 2850-2860, 2008.

[140] R. Bagatell, G. D. Paine-Murrieta, C. W. Taylor et al., "Induction of a heat shock factor 1-dependent stress response alters the cytotoxic activity of Hsp90-binding agents," Clinical Cancer Research, vol. 6, no. 8, pp. 3312-3318, 2000.

[141] A. K. McCollum, C. J. TenEyck, B. M. Sauer, D. O. Toft, and C. Erlichman, "Up-regulation of heat shock protein 27 induces resistance to 17-allylamino-demethoxygeldanamycin through a glutathione-mediated mechanism," Cancer Research, vol. 66, no. 22, pp. 10967-10975, 2006.

[142] A. K. McCollum, C. J. TenEyck, B. Stensgard et al., "Pglycoprotein-mediated resistance to Hsp90-directed therapy is eclipsed by the heat shock response," Cancer Research, vol. 68, no. 18, pp. 7419-7427, 2008.

[143] M. V. Powers, P. A. Clarke, and P. Workman, "Dual targeting of HSC70 and HSP72 inhibits HSP90 function and induces tumor-specific apoptosis," Cancer Cell, vol. 14, no. 3, pp. 250262, 2008.

[144] C. Dai, L. Whitesell, A. B. Rogers, and S. Lindquist, "Heat shock factor 1 is a powerful multifaceted modifier of carcinogenesis," Cell, vol. 130, no. 6, pp. 1005-1018, 2007.

[145] A. K. McCollum, K. B. Lukasiewicz, C. J. TenEyck, W. L. Lingle, D. O. Toft, and C. Erlichman, "Cisplatin abrogates the geldanamycin-induced heat shock response," Molecular Cancer Therapeutics, vol. 7, no. 10, pp. 3256-3264, 2008.

[146] J. H. Baek, Y. V. Liu, K. R. McDonald, J. B. Wesley, H. Zhang, and G. L. Semenza, "Spermidine/spermine N1acetyltransferase-1 binds to hypoxia-inducible factor- $1 \alpha$ $(\mathrm{HIF}-1 \alpha)$ and RACK1 and promotes ubiquitination and degradation of HIF-1 $\alpha$," Journal of Biological Chemistry, vol. 282, no. 46, pp. 33358-33366, 2007.

[147] Y. V. Liu, M. E. Hubbi, F. Pan et al., "Calcineurin promotes hypoxia-inducible factor $1 \alpha$ expression by dephosphorylating RACK1 and blocking RACK1 dimerization," Journal of Biological Chemistry, vol. 282, no. 51, pp. 37064-37073, 2007.

[148] E. S. Ehrlich, T. Wang, K. Luo et al., "Regulation of Hsp90 client proteins by a Cullin5-RING E3 ubiquitin ligase," Proceedings of the National Academy of Sciences of the United States of America, vol. 106, no. 48, pp. 20330-20335, 2010.
[149] M. J. Fay, K. A. Longo, G. A. Karathanasis et al., "Analysis of CUL-5 expression in breast epithelial cells, breast cancer cell lines, normal tissues and tumor tissues," Molecular Cancer, vol. 2, p. 40, 2003.

[150] C. Morrissey and R. L. Vessella, "The role of tumor microenvironment in prostate cancer bone metastasis," Journal of Cellular Biochemistry, vol. 101, no. 4, pp. 873-886, 2007.

[151] A. Orimo, P. B. Gupta, D. C. Sgroi et al., "Stromal fibroblasts present in invasive human breast carcinomas promote tumor growth and angiogenesis through elevated SDF-1/CXCL12 secretion," Cell, vol. 121, no. 3, pp. 335-348, 2005.

[152] P. A. Kenny, G. Y. Lee, and M. J. Bissell, “Targeting the tumor microenvironment," Frontiers in Bioscience, vol. 12, pp. 34683474, 2007.

[153] A. F. Olumi, P. Dazin, and T. D. Tlsty, "A novel coculture technique demonstrates that normal human prostatic fibroblasts contribute to tumor formation of LNCaP cells by retarding cell death," Cancer Research, vol. 58, no. 20, pp. 4525-4530, 1998.

[154] J. A. Tuxhorn, G. E. Ayala, M. J. Smith, V. C. Smith, T. D. Dang, and D. R. Rowley, "Reactive stroma in human prostate cancer: induction of myofibroblast phenotype and extracellular matrix remodeling," Clinical Cancer Research, vol. 8, no. 9, pp. 2912-2923, 2002.

[155] J. A. Tuxhorn, S. J. McAlhany, T. D. Dang, G. E. Ayala, and D. R. Rowley, "Stromal cells promote angiogenesis and growth of human prostate tumors in a differential reactive stroma (DRS) xenograft model," Cancer Research, vol. 62, no. 11, pp. 3298-3307, 2002.

[156] S. W. Hayward, Y. Wang, M. Cao et al., "Malignant transformation in a nontumorigenic human prostatic epithelial cell line," Cancer Research, vol. 61, no. 22, pp. 8135-8142, 2001.

[157] M. Hu, J. Yao, D. K. Carroll et al., "Regulation of in situ to invasive breast carcinoma transition," Cancer Cell, vol. 13, no. 5, pp. 394-406, 2008.

[158] M. Erkan, C. Reiser-Erkan, C. W. Michalski et al., "Cancerstellate cell interactions perpetuate the hypoxia-fibrosis cycle in pancreatic ductal adenocarcinoma," Neoplasia, vol. 11, no. 5, pp. 497-508, 2009.

[159] Y.-F. Shi, C.-C. Fong, Q. Zhang et al., "Hypoxia induces the activation of human hepatic stellate cells LX-2 through TGF$\beta$ signaling pathway," FEBS Letters, vol. 581, no. 2, pp. 203210, 2007.

[160] A. Masamune, K. Kikuta, T. Watanabe, K. Satoh, M. Hirota, and T. Shimosegawa, "Hypoxia stimulates pancreatic stellate cells to induce fibrosis and angiogenesis in pancreatic cancer," American Journal of Physiology, vol. 295, no. 4, pp. G709G717, 2008.

[161] J. A. Joyce, "Therapeutic targeting of the tumor microenvironment," Cancer Cell, vol. 7, no. 6, pp. 513-520, 2005.

[162] Y. Liu, X. M. Wen, E. L. H. Lui et al., "Therapeutic targeting of the PDGF and TGF- $\beta$-signaling pathways in hepatic stellate cells by PTK787/ZK22258," Laboratory Investigation, vol. 89, no. 10, pp. 1152-1160, 2009.

[163] Y. Liu, E. L. H. Lui, S. L. Friedman et al., "PTK787/ZK22258 attenuates stellate cell activation and hepatic fibrosis in vivo by inhibiting VEGF signaling," Laboratory Investigation, vol. 89, no. 2, pp. 209-221, 2009.

[164] S. Tugues, G. Fernandez-Varo, J. Muñoz-Luque et al., "Antiangiogenic treatment with sunitinib ameliorates inflammatory infiltrate, fibrosis, and portal pressure in cirrhotic rats," Hepatology, vol. 46, no. 6, pp. 1919-1926, 2007. 
[165] V. Gioni, T. Karampinas, G. Voutsinas et al., "Imatinib mesylate inhibits proliferation and exerts an antifibrotic effect in human breast stroma fibroblasts," Molecular Cancer Research, vol. 6, no. 5, pp. 706-714, 2008.

[166] X. Guo, H. Oshima, T. Kitmura, M. M. Taketo, and M. Oshima, "Stromal fibroblasts activated by tumor cells promote angiogenesis in mouse gastric cancer," Journal of Biological Chemistry, vol. 283, no. 28, pp. 19864-19871, 2008.

[167] S. J. Myung, J.-H. Yoon, B. H. Kim, J.-H. Lee, E. U. Jung, and H.-S. Lee, "Heat shock protein 90 inhibitor induces apoptosis and attenuates activation of hepatic stellate cells," Journal of Pharmacology and Experimental Therapeutics, vol. 330, no. 1, pp. 276-282, 2009.

[168] G. Bergers and S. Song, "The role of pericytes in blood-vessel formation and maintenance," Neuro-Oncology, vol. 7, no. 4, pp. 452-464, 2005.

[169] N. Reinmuth, W. Liu, Y. D. Jung et al., "Induction of VEGF in perivascular cells defines a potential paracrine mechanism for endothelial cell survival," FASEB Journal, vol. 15, no. 7, pp. 1239-1241, 2001.

[170] S. Song, A. J. Ewald, W. Stallcup, Z. Werb, and G. Bergers, "PDGFR $\beta+$ perivascular progenitor cells in tumours regulate pericyte differentiation and vascular survival," Nature Cell Biology, vol. 7, no. 9, pp. 870-879, 2005.

[171] K. Pietras, J. Pahler, G. Bergers, and D. Hanahan, "Functions of paracrine PDGF signaling in the proangiogenic tumor stroma revealed by pharmacological targeting," PLoS Medicine, vol. 5, no. 1, article e19, 2008.

[172] A. Kamal, L. Thao, J. Sensintaffar et al., "A high-affinity conformation of Hsp90 confers tumour selectivity on Hsp90 inhibitors," Nature, vol. 425, no. 6956, pp. 407-410, 2003.

[173] G. Chiosis, H. Huezo, N. Rosen, E. Mimnaugh, L. Whitesell, and L. Neckers, "17AAG: low target binding affinity and potent cell activity-finding an explanation," Molecular Cancer Therapeutics, vol. 2, no. 2, pp. 123-129, 2003.

[174] D. C. Radisky, "Fibroblasts act as co-conspirators for chemotherapy resistance," Cancer Biology and Therapy, vol. 7, no. 9, pp. 1348-1349, 2008.

[175] Y. Crawford, I. Kasman, L. Yu et al., "PDGF-C mediates the angiogenic and tumorigenic properties of fibroblasts associated with tumors refractory to anti-VEGF treatment," Cancer Cell, vol. 15, no. 1, pp. 21-34, 2009.

[176] J. J. Virrey, E. B. Golden, W. Sivakumar et al., "Gliomaassociated endothelial cells are chemoresistant to temozolomide," Journal of Neuro-Oncology, vol. 95, no. 1, pp. 13-22, 2009.

[177] G. Bergers, S. Song, N. Meyer-Morse, E. Bergsland, and D. Hanahan, "Benefits of targeting both pericytes and endothelial cells in the tumor vasculature with kinase inhibitors," Journal of Clinical Investigation, vol. 111, no. 9, pp. 12871295, 2003.

[178] K. E. Hovinga, L. J. Stalpers, C. van Bree, et al., "Radiationenhanced vascular endothelial growth factor (VEGF) secretion in glioblastoma multiforme cell lines-a clue to radioresistance?" Journal of Neuro-Oncology, vol. 74, no. 2, pp. 99103, 2005.

[179] L. W. Chung, W. C. Huang, S. Y. Sung et al., "Stromalepithelial interaction in prostate cancer progression," Clinical Genitourinary Cancer, vol. 5, no. 2, pp. 162-170, 2006.

[180] N. Kamochi, M. Nakashima, S. Aoki et al., "Irradiated fibroblast-induced bystander effects on invasive growth of squamous cell carcinoma under cancer-stromal cell interaction," Cancer Science, vol. 99, no. 12, pp. 2417-2427, 2008.
[181] B. J. Moeller, Y. Cao, C. Y. Li, and M. W. Dewhirst, "Radiation activates HIF-1 to regulate vascular radiosensitivity in tumors: role of reoxygenation, free radicals, and stress granules," Cancer Cell, vol. 5, no. 5, pp. 429-441, 2004.

[182] B. J. Moeller, M. R. Dreher, Z. N. Rabbani et al., "Pleiotropic effects of HIF-1 blockade on tumor radiosensitivity," Cancer Cell, vol. 8, no. 2, pp. 99-110, 2005.

[183] J. Brieger, J. Kattwinkel, M. Berres, J. Gosepath, and W. J. Mann, "Impact of vascular endothelial growth factor release on radiation resistance," Oncology Reports, vol. 18, no. 6, pp. 1597-1601, 2007.

[184] R. F. Hwang, T. Moore, T. Arumugam et al., "Cancerassociated stromal fibroblasts promote pancreatic tumor progression," Cancer Research, vol. 68, no. 3, pp. 918-926, 2008.

[185] K. K. C. Tsai, J. Stuart, Y.-Y.E. Chuang, J. B. Little, and Z.M. Yuan, "Low-dose radiation-induced senescent stromal fibroblasts render nearby breast cancer cells radioresistant," Radiation Research, vol. 172, no. 3, pp. 306-313, 2009.

[186] M. Li, G. Ping, C. Plathow et al., "Small molecule receptor tyrosine kinase inhibitor of platelet-derived growth factor signaling (SU9518) modifies radiation response in fibroblasts and endothelial cells," BMC Cancer, vol. 6, article 79, 2006.

[187] K. S. Bisht, C. M. Bradbury, D. Mattson et al., "Geldanamycin and 17-allylamino-17-demethoxygeldanamycin potentiate the in vitro and in vivo radiation response of cervical tumor cells via the heat shock protein 90-mediated intracellular signaling and cytotoxicity," Cancer Research, vol. 63, no. 24, pp. 8984-8995, 2003.

[188] J. S. Russell, W. Burgan, K. A. Oswald, K. Camphausen, and P. J. Tofilon, "Enhanced cell killing induced by the combination of radiation and the heat shock protein 90 inhibitor 17-allylamino-17-demethoxygeldanamycin: a multitarget approach to radiosensitization," Clinical Cancer Research, vol. 9, no. 10, pp. 3749-3755, 2003.

[189] X. Yin, H. Zhang, K. Lundgren, L. Wilson, F. Burrows, and C. G. Shores, "BIIB021, a novel Hsp90 inhibitor, sensitizes head and neck squamous cell carcinoma to radiotherapy," International Journal of Cancer, vol. 126, no. 5, pp. 12161225,2010

[190] D. L. Schwartz, G. Powis, A. Thitai-Kumar et al., "The selective hypoxia inducible factor-1 inhibitor PX-478 provides in vivo radiosensitization through tumor stromal effects," Molecular Cancer Therapeutics, vol. 8, no. 4, pp. 947-958, 2009.

[191] A. E. Kabakov, Y. M. Makarova, and Y. V. Malyutina, "Radiosensitization of human vascular endothelial cells through Hsp90 inhibition with 17-N-allilamino-17demethoxygeldanamycin," International Journal of Radiation Oncology Biology Physics, vol. 71, no. 3, pp. 858-865, 2008.

[192] F. Winkler, S. V. Kozin, R. T. Tong et al., "Kinetics of vascular normalization by VEGFR2 blockade governs brain tumor response to radiation: role of oxygenation, angiopoietin-1, and matrix metalloproteinases," Cancer Cell, vol. 6, no. 6, pp. 553-563, 2004.

[193] P. J. Mishra, P. J. Mishra, R. Humeniuk et al., "Carcinomaassociated fibroblast-like differentiation of human mesenchymal stem cells," Cancer Research, vol. 68, no. 11, pp. 4331-4339, 2008.

[194] R. S. Kerbel, R. Benezra, D. C. Lyden et al., "Endothelial progenitor cells are cellular hubs essential for neoangiogenesis of certain aggressive adenocarcinomas and metastatic transition but not adenomas," Proceedings of the National Academy of 
Sciences of the United States of America, vol. 105, no. 34, p. E54, 2008, author reply E55.

[195] D. K. Jin, K. Shido, H.-G. Kopp et al., "Cytokine-mediated deployment of SDF-1 induces revascularization through recruitment of CXCR4+ hemangiocytes," Nature Medicine, vol. 12, no. 5, pp. 557-567, 2006.

[196] S. Rey, K. Lee, C. J. Wang et al., "Synergistic effect of HIF- $1 \alpha$ gene therapy and HIF-1-activated bone marrowderived angiogenic cells in a mouse model of limb ischemia," Proceedings of the National Academy of Sciences of the United States of America, vol. 106, no. 48, pp. 20399-20404, 2010.

[197] G. Tabatabai, B. Frank, R. Möhle, M. Weller, and W. Wick, "Irradiation and hypoxia promote homing of haematopoietic progenitor cells towards gliomas by TGF- $\beta$-dependent HIF$1 \alpha$-mediated induction of CXCL12," Brain, vol. 129, no. 9, pp. 2426-2435, 2006.

[198] M. Pàez-Ribes, E. Allen, J. Hudock et al., "Antiangiogenic therapy elicits malignant progression of tumors to increased local invasion and distant metastasis," Cancer Cell, vol. 15, no. 3, pp. 220-231, 2009.

[199] F. Shojaei, X. Wu, A. K. Malik et al., "Tumor refractoriness to anti-VEGF treatment is mediated by $\mathrm{CD} 11 \mathrm{~b}^{+} \mathrm{Gr} 1^{+}$myeloid cells," Nature Biotechnology, vol. 25, no. 8, pp. 911-920, 2007.

[200] R. Du, K. V. Lu, C. Petritsch et al., "HIF1 $\alpha$ induces the recruitment of bone marrow-derived vascular modulatory cells to regulate tumor angiogenesis and invasion," Cancer Cell, vol. 13, no. 3, pp. 206-220, 2008.

[201] F. Shojaei, X. Wu, C. Zhong et al., "Bv8 regulates myeloidcell-dependent tumour angiogenesis," Nature, vol. 450, no. 7171, pp. 825-831, 2007.

[202] B. Weigelt, A. T. Lo, C. C. Park, J. W. Gray, and M. J. Bissell, "HER2 signaling pathway activation and response of breast cancer cells to HER2-targeting agents is dependent strongly on the 3D microenvironment," Breast Cancer Research and Treatment, vol. 122, no. 1, pp. 35-43, 2010.

[203] A. Mammoto, K. M. Connor, T. Mammoto et al., "A mechanosensitive transcriptional mechanism that controls angiogenesis," Nature, vol. 457, no. 7233, pp. 1103-1108, 2009.

[204] O. Ayrault, M. D. Godeny, C. Dillon et al., "Inhibition of Hsp90 via 17-DMAG induces apoptosis in a p53-dependent manner to prevent medulloblastoma," Proceedings of the National Academy of Sciences of the United States of America, vol. 106, no. 40, pp. 17037-17042, 2009.

[205] E. Caldas-Lopes, L. Cerchietti, J. H. Ahn et al., "Hsp90 inhibitor PU-H71, a multimodal inhibitor of malignancy, induces complete responses in triple-negative breast cancer models," Proceedings of the National Academy of Sciences of the United States of America, vol. 106, no. 20, pp. 8368-8373, 2009.

[206] E. D. Pleasance, R. K. Cheetham, P. J. Stephens et al., "A comprehensive catalogue of somatic mutations from a human cancer genome," Nature, vol. 463, no. 7278, pp. 191196, 2010.

[207] E. D. Pleasance, P. J. Stephens, S. O’Meara et al., “A smallcell lung cancer genome with complex signatures of tobacco exposure," Nature, vol. 463, no. 7278, pp. 184-190, 2010.

[208] W. Arap, M. G. Kolonin, M. Trepel et al., "Steps toward mapping the human vasculature by phage display," Nature Medicine, vol. 8, no. 2, pp. 121-127, 2002.

[209] J. A. Joyce, P. Laakkonen, M. Bernasconi, G. Bergers, E. Ruoslahti, and D. Hanahan, "Stage-specific vascular markers revealed by phage display in a mouse model of pancreatic islet tumorigenesis," Cancer Cell, vol. 4, no. 5, pp. 393-403, 2003.
[210] J. A. Hoffman, E. Giraudo, M. Singh et al., "Progressive vascular changes in a transgenic mouse model of squamous cell carcinoma," Cancer Cell, vol. 4, no. 5, pp. 383-391, 2003.

[211] P. Olson, J. Lu, H. Zhang et al., "MicroRNA dynamics in the stages of tumorigenesis correlate with hallmark capabilities of cancer," Genes and Development, vol. 23, no. 18, pp. 21522165, 2009.

[212] D. Song, R. Chaerkady, A. C. Tan et al., "Antitumor activity and molecular effects of the novel heat shock protein 90 inhibitor, IPI-504, in pancreatic cancer," Molecular Cancer Therapeutics, vol. 7, no. 10, pp. 3275-3284, 2008.

[213] E. A. Eisenhauer, P. Therasse, J. Bogaerts et al., "New response evaluation criteria in solid tumours: revised RECIST guideline (version 1.1)," European Journal of Cancer, vol. 45, no. 2, pp. 228-247, 2009.

[214] D. B. Solit, I. Osman, D. Polsky et al., "Phase II trial of 17-allylamino-17-demethoxygeldanamycin in patients with metastatic melanoma," Clinical Cancer Research, vol. 14, no. 24, pp. 8302-8307, 2008.

[215] W. Cai, A. Ebrahimnejad, K. Chen et al., "Quantitative radioimmunoPET imaging of EphA2 in tumor-bearing mice," European Journal of Nuclear Medicine and Molecular Imaging, vol. 34, no. 12, pp. 2024-2036, 2007.

[216] P. M. Smith-Jones, D. B. Solit, T. Akhurst, F. Afroze, N. Rosen, and S. M. Larson, "Imaging the pharmacodynamics of HER2 degradation in response to Hsp90 inhibitors," Nature Biotechnology, vol. 22, no. 6, pp. 701-706, 2004.

[217] T. T. Batchelor, A. G. Sorensen, E. di Tomaso et al., "AZD2171, a pan-VEGF receptor tyrosine kinase inhibitor, normalizes tumor vasculature and alleviates edema in glioblastoma patients," Cancer Cell, vol. 11, no. 1, pp. 83-95, 2007.

[218] L. Xu, D. G. Duda, E. di Tomaso, et al., "Direct evidence that bevacizumab, an anti-VEGF antibody, up-regulates SDF1alpha, CXCR4, CXCL6, and neuropilin 1 in tumors from patients with rectal cancer," Cancer Research, vol. 69, no. 20, pp. 7905-7910, 2009. 


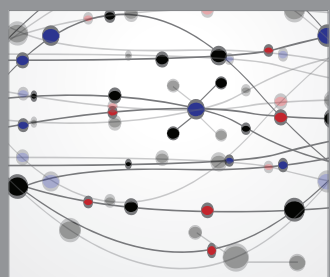

The Scientific World Journal
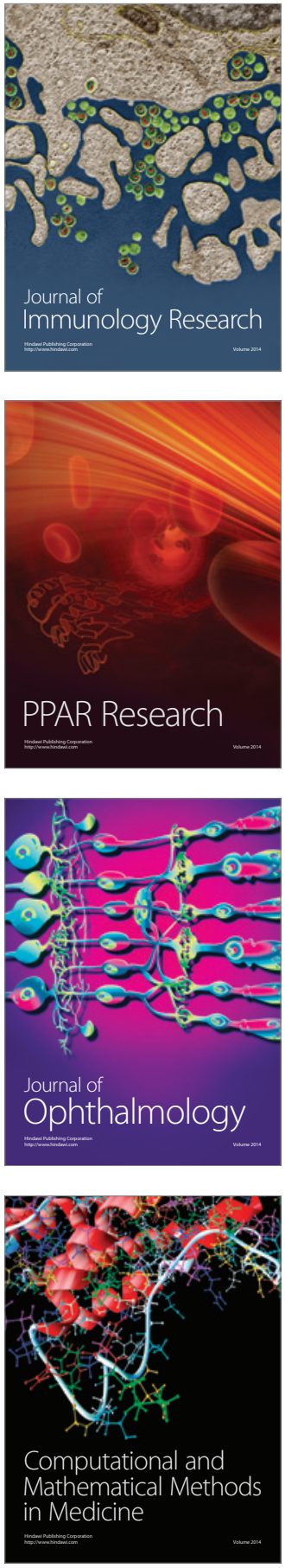

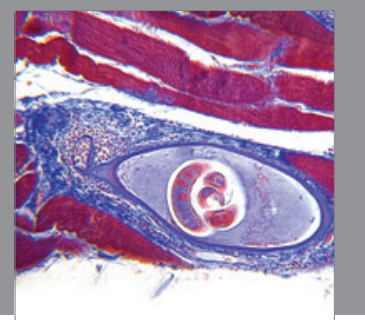

Gastroenterology

Research and Practice
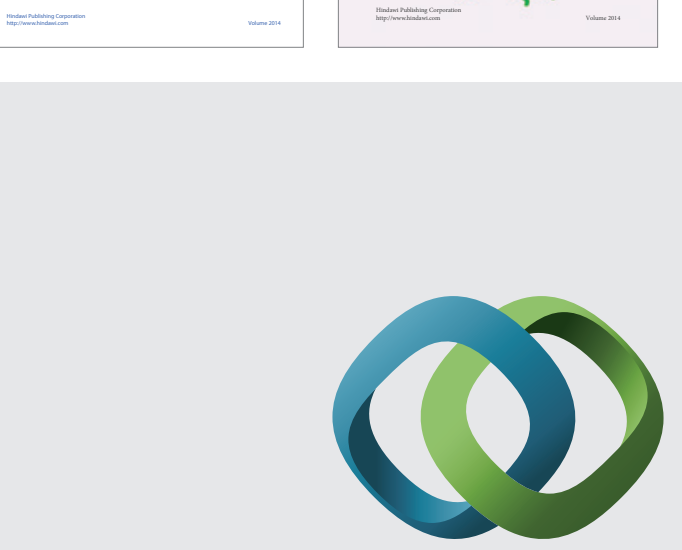

\section{Hindawi}

Submit your manuscripts at

http://www.hindawi.com
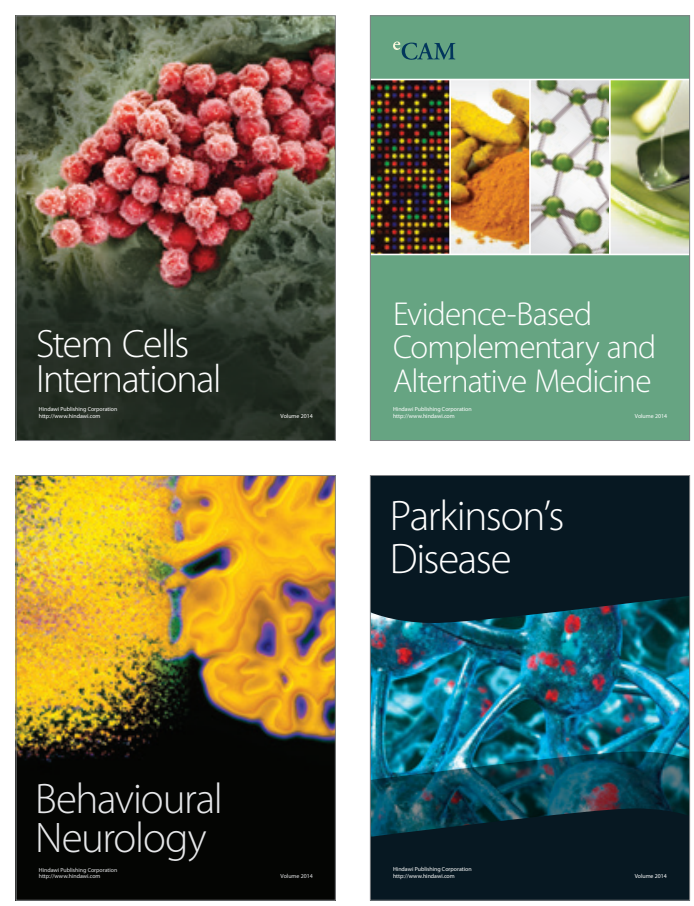

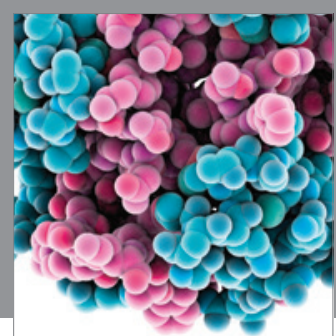

Journal of
Diabetes Research

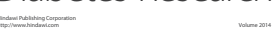

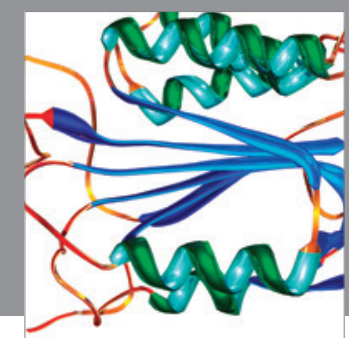

Disease Markers
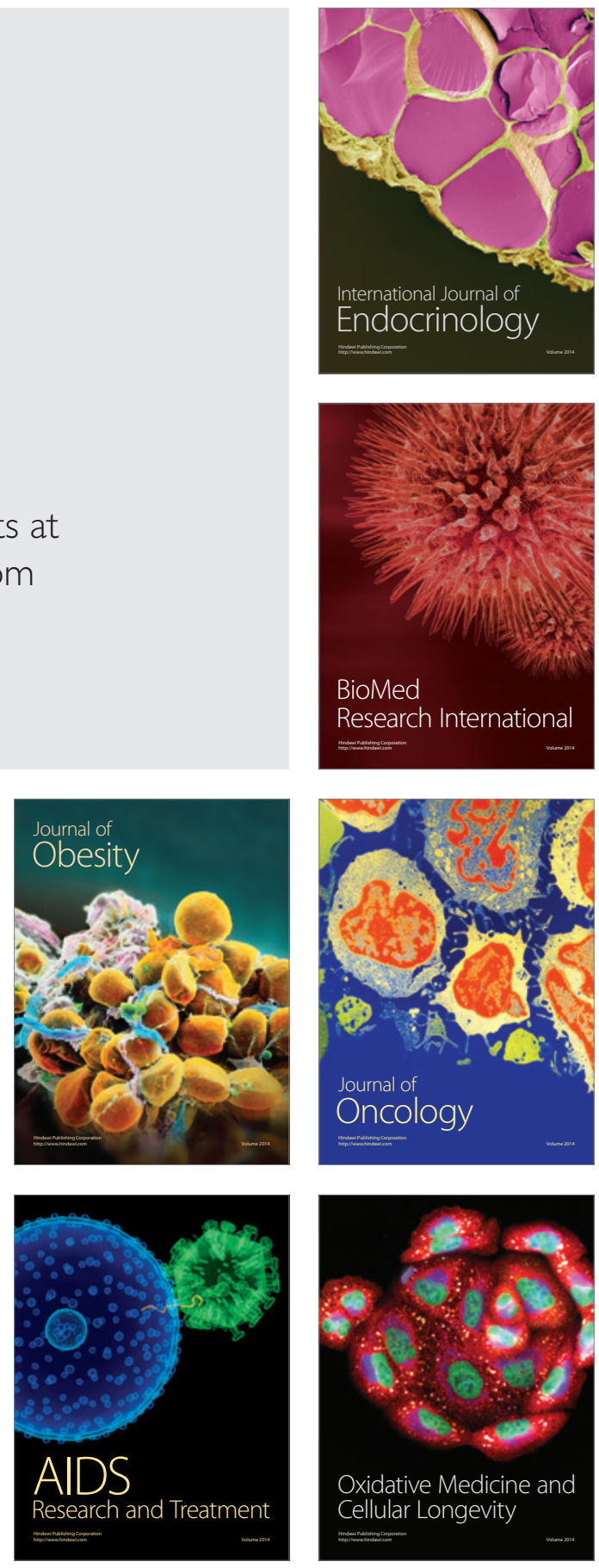